\title{
COMPLEX STRUCTURE IN CLASS 0 PROTOSTELLAR ENVELOPES. III. VELOCITY GRADIENTS IN NON-AXISYMMETRIC ENVELOPES, INFALL, OR ROTATION?**
}

\author{
John J. Tobin ${ }^{1,6}$, Lee Hartmann ${ }^{1}$, Edwin Bergin ${ }^{1}$, Hsin-Fang Chiang $^{2}$, Leslie W. Looney ${ }^{2}$, Claire J. Chandler $^{3}$, \\ SÉBASTIEN MARET ${ }^{4}$, AND FABIAN Heitsch ${ }^{5}$ \\ ${ }^{1}$ Department of Astronomy, University of Michigan, Ann Arbor, MI 48109, USA; jtobin@nrao.edu \\ 2 Department of Astronomy, University of Illinois at Champaign/Urbana, Urbana, IL 61801, USA \\ ${ }^{3}$ National Radio Astronomy Observatory, P.O. Box O, Socorro, NM 87801, USA \\ ${ }^{4}$ UJF-Grenoble 1/CNRS-INSU, Institut de Planétologie et d'Astrophysique de Grenoble (IPAG) UMR 5274, Grenoble F-38041, France \\ ${ }^{5}$ Department of Astronomy, University of North Carolina, Chapel Hill, NC, USA \\ Received 2011 November 23; accepted 2012 January 9; published 2012 February 29
}

\begin{abstract}
We present an interferometric kinematic study of morphologically complex protostellar envelopes based on observations of the dense gas tracers $\mathrm{N}_{2} \mathrm{H}^{+}$and $\mathrm{NH}_{3}$. The strong asymmetric nature of most envelopes in our sample leads us to question the common interpretation of velocity gradients as rotation, given the possibility of projection effects in the observed velocities. Several "idealized" sources with well-ordered velocity fields and envelope structures are now analyzed in more detail. We compare the interferometric data to position-velocity (PV) diagrams of kinematic models for spherical rotating collapse and filamentary rotating collapse. For this purpose, we developed a filamentary parameterization of the rotating collapse model to explore the effects of geometric projection on the observed velocity structures. We find that most envelopes in our sample have PV structures that can be reproduced by an infalling filamentary envelope projected at different angles within the plane of the sky. The infalling filament produces velocity shifts across the envelope that can mimic rotation, especially when viewed at single-dish resolutions and the axisymmetric rotating collapse model does not uniquely describe any data set. Furthermore, if the velocities are assumed to reflect rotation, then the inferred centrifugal radii are quite large in most cases, indicating significant fragmentation potential or more likely another component to the line-center velocity. We conclude that ordered velocity gradients cannot be interpreted as rotation alone when envelopes are non-axisymmetric and that projected infall velocities likely dominate the velocity field on scales larger than 1000 AU.
\end{abstract}

Key words: ISM: kinematics and dynamics - ISM: molecules - radio lines: ISM - stars: formation

Online-only material: color figures

\section{INTRODUCTION}

The angular momentum of protostellar cores and envelopes plays an important role in star formation. To the extent that the rotation of the protostellar cloud is non-negligible, a significant fraction of the star's final mass must be accreted through a circumstellar disk (Zhu et al. 2010; Kratter et al. 2010). In addition, collapse with large amounts of angular momenta can result in gravitational fragmentation into companion stars (Burkert \& Bodenheimer 1993; Boss 1995, and references therein). Finally, differing initial angular momenta of clouds are likely to produce a distribution of binary separations and differing initial disk configurations, with implications for planet formation. Thus, the importance of angular momentum in the star and planet formation process illustrates the need to characterize its magnitude in collapsing protostellar cores.

Observational attempts have been made to characterize the rotation of dense cores and protostellar envelopes on scales of $0.05-0.5 \mathrm{pc}$, principally using the dense gas tracers $\mathrm{NH}_{3}$ and $\mathrm{N}_{2} \mathrm{H}^{+}$(Goodman et al. 1993; Caselli et al. 2002). Velocity gradients are clearly detected in most objects, and observations with increasing spatial resolution tend to find larger velocity

\footnotetext{
* Based on observations carried out with the IRAM Plateau de Bure Interferometer, Combined Array for Research in Millimeter-wave Astronomy (CARMA), and the NRAO Very Large Array.

6 Current address: Hubble Fellow, National Radio Astronomy Observatory, Charlottesville, VA 22903, USA.
}

gradients on the same spatial scales owing to less smoothing of the velocity field. On smaller scales, Volgenau et al. (2006) and Chen et al. (2007) examined the kinematic structures of protostellar envelopes down to $\sim 1000 \mathrm{AU}$ scales, only finding clear evidence of rotation in a few cases. The detected velocity gradients are often interpreted under the assumption of solidbody rotation, even in protostellar systems; this enables simple calculations of angular momentum (Goodman et al. 1993; Chen et al. 2007; Chiang et al. 2010; Tanner \& Arce 2011). Several studies have also found the possible signatures of rotation on sub-1000 AU scales from high-velocity molecular line wings in interferometric data (Brinch et al. 2007; Lee et al. 2009).

In addition to rotation, the velocity fields of protostellar envelopes will necessarily include infall motions. Initial models of collapsing protostellar envelopes assumed spherical geometry (Larson 1969; Shu 1977); later models included angular momentum, which leads to flattening of the envelope on small scales near the forming disk (Cassen \& Moosman 1981; Terebey et al. 1984). Infall is typically probed via single-dish spectra, and the blueshifted asymmetry of an optically thick line profile (e.g., $\mathrm{HCO}^{+} \mathrm{HCN}$, and CS) is the classic signature of collapse (Walker et al. 1986; Zhou 1992; Zhou et al. 1993). The insideout collapse model (Shu 1977) is typically adopted to describe the velocity field and coupled with radiative transfer modeling to derive infall rates and infall radii (Zhou et al. 1993; Ward-Thompson \& Buckley 2001; Narayanan et al. 2002). The derived infall radii are often on the order of $\sim 5000 \mathrm{AU}$, 
Table 1

Source Properties

\begin{tabular}{|c|c|c|c|c|c|c|c|c|c|c|}
\hline Source & $\begin{array}{l}\text { R.A. } \\
(\mathrm{J} 2000)\end{array}$ & $\begin{array}{c}\text { Decl. } \\
(\mathrm{J} 2000)\end{array}$ & $\begin{array}{l}\text { Distance } \\
\quad(\mathrm{pc})\end{array}$ & $\begin{array}{c}\operatorname{Mass}_{8 \mu \mathrm{m}} \\
\left(M_{\odot}\right) \\
(r<0.05 \mathrm{pc})\end{array}$ & $\begin{array}{l}\text { Mass }_{\text {submm }^{\mathrm{a}}} \\
\quad\left(M_{\odot}\right)\end{array}$ & $\begin{array}{l}L_{\text {bol }} \\
\left(L_{\odot}\right)\end{array}$ & $\begin{array}{l}T_{\text {bol }} \\
(\mathrm{K})\end{array}$ & $\begin{array}{l}\text { Morphological } \\
\text { Classification }\end{array}$ & $\begin{array}{l}\text { Outflow PA } \\
\left({ }^{\circ}\right)\end{array}$ & $\begin{array}{c}\text { References } \\
\left.\text { (Dist., } M_{\text {ref }}, L_{\text {bol }}, T_{\text {bol }}\right)\end{array}$ \\
\hline L1157 & $20: 39: 06.25$ & $+68: 02: 15.9$ & 300 & 2.7 & 2.2 & 3.0 & 29 & Flattened & 150 & $4,3,2,9$ \\
\hline L1165 & $22: 06: 50.46$ & $+59: 02: 45.9$ & 300 & 1.1 & 0.32 & 13.9 & 46 & Irregular & 225 & $4,6,6,1$ \\
\hline CB 230 & $21: 17: 38.56$ & $+68: 17: 33.3$ & 300 & 1.1 & 1.1 & 7.2 & 69 & One-sided & 0 & $4,8,2,9$ \\
\hline HH 211 & $03: 43: 56.78$ & $+32: 00: 49.8$ & 230 & 1.1 & 1.5 & 3.02 & 24 & Irregular & 116 & $12,2,2,2,5$ \\
\hline IRAS $16253-2429$ & $16: 28: 21.42$ & $-24: 36: 22.1$ & 125 & 0.8 & 0.98 & 0.25 & 35 & Spheroidal & 20 & $7,10,2,2,11$ \\
\hline
\end{tabular}

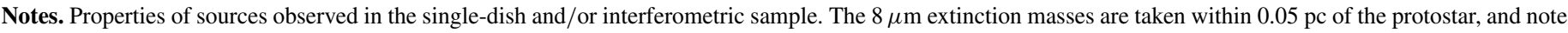

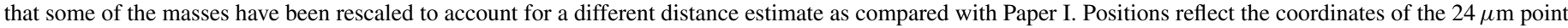
source from Spitzer data or the $3 \mathrm{~mm}$ continuum position for protostars observed with CARMA.

a Mass was computed with sub/millimeter bolometer data assuming an isothermal temperature.

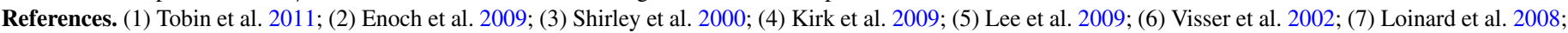
(8) Kauffmann et al. 2008; (9) Froebrich 2005; (10) Young et al. 2006; (11) Arce \& Sargent 2006; (12) Hirota et al. 2011.

indicating that material is likely falling in from relatively large radii. Inside the infall radius, the envelope is expected to exhibit a differential rotation curve, owing to the conservation of angular momentum; the outer core is often assumed to have solidbody rotation (Terebey et al. 1984). However, Ward-Thompson \& Buckley (2001) noted that one of their models required inward motions of the envelope out to its boundary, indicating that inside-out collapse may not be realistic and an "outside-in" collapse may be more appropriate in some cases (e.g., Foster \& Chevalier 1993).

Contrary to the assumptions of early models, simulations of star formation in turbulent clouds indicate that infall to protostars is generally complex, non-axisymmetric, and often filamentary (e.g., Bate et al. 2003; Smith et al. 2011). Indeed, observations at higher spatial resolution show that most preand protostellar cores are elongated (Benson \& Myers 1989; Myers et al. 1991; Bacmann et al. 2000; Stutz et al. 2009) and more likely to be prolate (Ryden 1996; Myers 2005). In the first paper of this series (Tobin et al. 2010, hereafter Paper I), we used Spitzer Infrared Array Camera (IRAC) images of a set of Class 0 protostars to characterize dense envelope structures in extinction at $8 \mu \mathrm{m}$; the results showed that many systems have very flattened, filamentary, and often strongly non-axisymmetric density structures.

The recognition of the complexity and non-axisymmetry of many protostellar envelopes poses substantial problems for any attempt to infer angular momenta and the interpretation of line profiles under the assumption of spherical collapse. Kinematic observations necessarily refer to integrations along the line of sight of structures with uncertain geometry, and because only one of the three velocity components is detected, there is substantial ambiguity in interpreting the observations as rotation or infall (e.g., Dib et al. 2010; R. J. Smith et al. 2012, in preparation). More specifically, infall (or outflow) could masquerade as rotation, depending on the geometry and viewing aspect of the envelopes.

In the second paper of this series (Tobin et al. 2011, hereafter Paper II), we presented observations of the Class 0 envelopes of Paper $\mathrm{I}$ in the dense molecular tracers $\mathrm{N}_{2} \mathrm{H}^{+}$and $\mathrm{NH}_{3}$. Using moment maps, we showed that the kinematics of these envelopes, like their density structures, were often complex and difficult to interpret. The interferometric data presented in Tobin et al. (2011) had a higher frequency of envelopes with velocity gradients that could be interpreted as rotation, as compared with Volgenau et al. (2006) and Chen et al. (2007); however, we were not convinced that our data were clearly tracing a rotation signature, even in ideal cases.

In this paper, we proceed to a more detailed analysis of the kinematics for a subset of the sample from Papers I and II where the velocity fields appear to be rather well ordered. Mindful of the interpretation difficulties in complex geometry, we develop a simple kinematic model for the collapse of a filament to compare with the observations. While significant ambiguities remain, we find evidence for rotation in some systems on small ( $<1000 \mathrm{AU}$ ) scales but infer that infall motions are likely to dominate the radial velocity structure on scales $>1000 \mathrm{AU}$. Our findings emphasize the importance of following the kinematics to the smallest scales to determine the angular momenta of infalling envelopes and the need for further, more realistic simulations of the large-scale velocity fields in star-forming regions for comparison with observations.

This paper is organized as follows: Section 2 briefly describes the models of filament and axisymmetric collapse, Section 3 describes the comparison of the models with the observed channel maps and position-velocity (PV) diagrams, and Section 4 discusses our results. The observational details were described in Paper II and are omitted here for brevity. We will present data for five sources, listed in Table 1, four of which were observed in the $\mathrm{N}_{2} \mathrm{H}^{+}$molecule (one with the Plateau de Bure Interferometer and three with Combined Array for Research in Millimeterwave Astronomy, CARMA), and one source was observed in $\mathrm{NH}_{3}$ with the Very Large Array. ${ }^{7}$

\section{KINEMATIC MODELS}

Most previous work on the velocity structure of protostellar envelopes assumed spherical symmetry or axisymmetry in the interpretation of kinematic data. However, in the absence of symmetry, the physical interpretation of velocity gradients is now non-trivial. The difficulties of interpreting velocity fields of protostellar envelopes seen in projection are schematically illustrated in Figure 1. For simplicity we plot only the limiting radial velocities expected from the emitting envelope. In the case of spherical symmetry (or axisymmetry in the appropriate plane), pure uniform rotation (solid-body) directly yields a simple linear velocity gradient across the envelope; the velocity spread is due to the superposition of velocity components at

\footnotetext{
7 The National Radio Astronomy Observatory is a facility of the National Science Foundation operated under cooperative agreement by Associated Universities, Inc.
} 


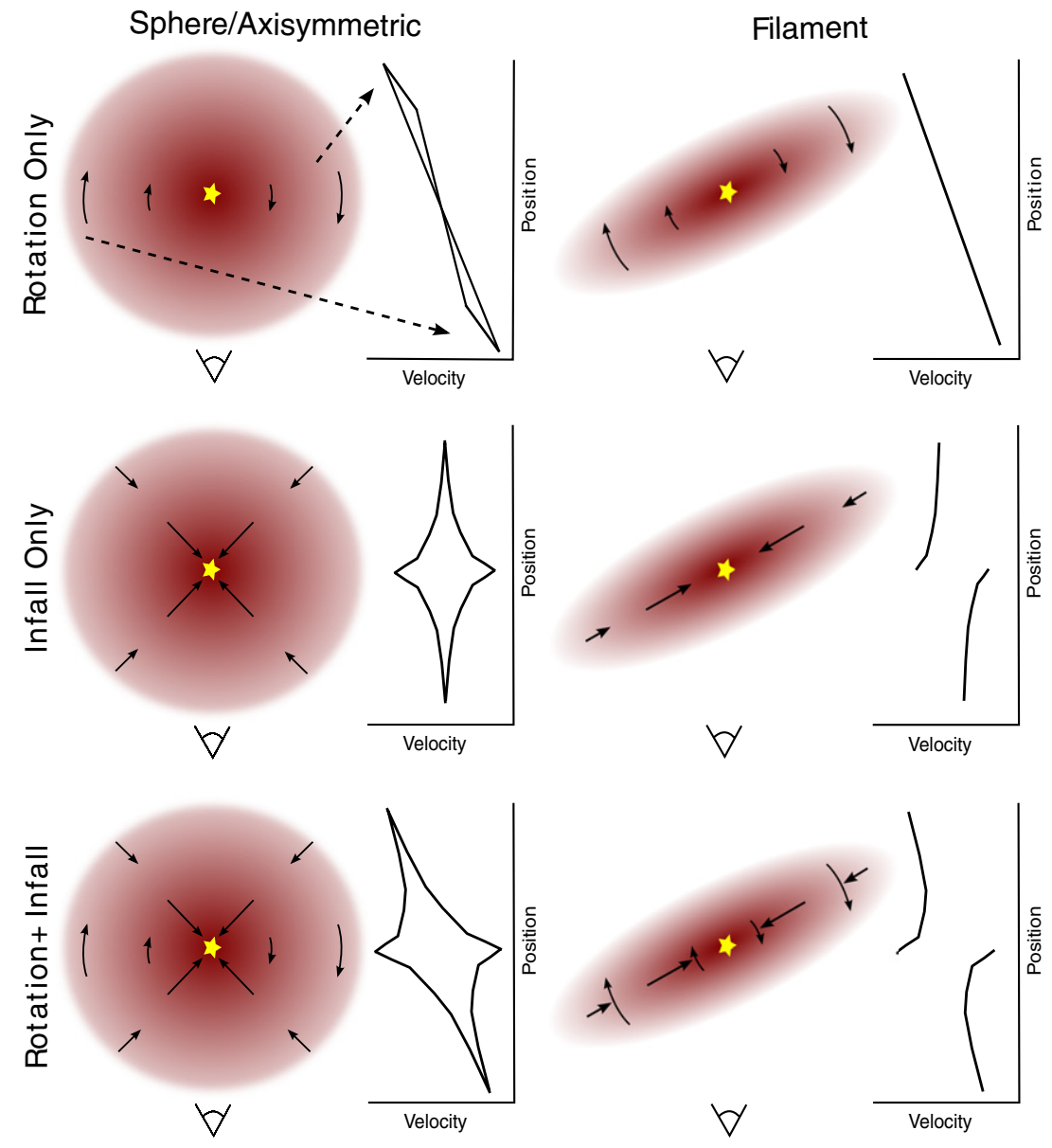



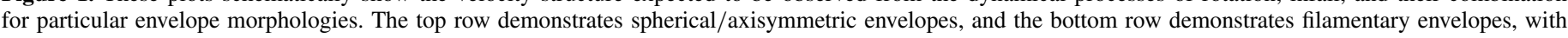

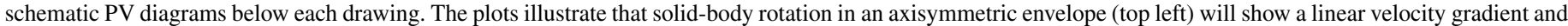

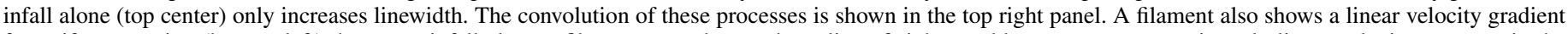

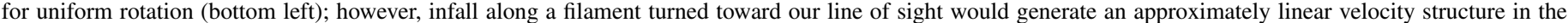
absence of rotation (bottom middle). When these processes are convolved in the bottom right panel, the two processes are difficult to separate.

(A color version of this figure is available in the online journal.)

intermediate scales and disappears toward the envelope edge. Pure radial infall yields no systematic gradient across the envelope, but an increase in the velocity dispersion near the central gravitating mass. In general, we expect both types of motions to be present, resulting in the skewed velocity caustics shown in the upper right. Note that a Keplerian rotation curve is distinct from that of solid-body rotation and appears exactly like the radial infall curve.

Proceeding to a filamentary envelope, the simplest of nonaxisymmetric structures, immediately introduces a fundamental ambiguity, as both pure solid-body rotation and pure infall can yield similar velocity gradients from one side of the envelope to the other (bottom two panels). Including both types of motion makes the situation even more uncertain. As the observations clearly indicate that non-axisymmetric or filamentary envelopes are common (Paper I), this poses a serious problem for interpretation.

In principle, the emission in PV diagrams, such as indicated schematically here, can provide some indication of true filamentary or non-axisymmetric structure. For instance, axisymmetric envelopes (especially those infalling) will tend to have larger velocity dispersions at a given position because material along the line of sight will have differing velocity vectors than in a highly linear structure. In addition, material falling in close to the protostar will tend to yield both redshifted and blueshifted emission at each position, whereas a filament will exhibit more of an abrupt change from red to blue across the position of the protostar (bottom right panels of Figure 1).

\subsection{Analytic Model of Filamentary and Axisymmetric Collapse}

Given the complications introduced to the kinematics by non-axisymmetric systems, we make an initial step toward interpreting the observations by developing a simple kinematic model for comparison with PV diagrams. We have modified the rotating collapse model (Ulrich 1976; Cassen \& Moosman 1981, hereafter CMU model), which has been extensively used in studies of protostellar envelopes. The infalling gas in the CMU model is assumed to fall in from an initial spherical cloud rotating with constant angular velocity. With this assumption, the streamlines of the infalling gas do not intersect, and thus the (supersonic) motion can be considered in the limit of ballistic trajectories around a central gravitating mass. (This neglects the self-gravity of the envelope; but Terebey et al. (1984) showed that the CMU model could be taken as the inner region of a more general model of self-gravitating spherical cloud collapse.)

By neglecting the self-gravity of the infalling gas, we can adopt the axisymmetric velocity field of the CMU solution. 
We create a filament by modifying the density distribution such that all the infalling gas is confined within specified streamlines spanning $\Delta \phi=30^{\circ}$ in azimuth on opposite sides of the envelope (mirror symmetry). This filamentary geometry enables us to project the density distribution of the envelope to different orientations in the sky in order to examine the effects of projection on the kinematic structure. The height of the envelope in the $z$-direction is taken to be comparable to the thickness of the filament as viewed from top-down but does not play a crucial role in the observed kinematic structure.

The streamlines composing the filament correspond to limits in velocity space for emission at a given position, producing curves that can be compared with observed PV diagrams, a similar concept to that of using "caustics" to analyze position-redshift diagrams of galaxy clusters (e.g., Regos \& Geller 1989). The velocity components of the infalling, rotating gas are given by (Ulrich 1976)

$$
\begin{gathered}
v_{r}=-\left(\frac{G M}{r}\right)^{1 / 2}\left(1+\frac{\cos \theta}{\cos \theta_{0}}\right)^{1 / 2}, \\
v_{\phi}=\left(\frac{G M}{r}\right)^{1 / 2}\left(1-\frac{\cos \theta}{\cos \theta_{0}}\right)^{1 / 2}\left(\frac{\sin \theta_{0}}{\sin \theta}\right), \\
v_{\theta}=\left(\frac{G M}{r}\right)^{1 / 2}\left(\cos \theta_{0}-\cos \theta\right)\left(\frac{\cos \theta_{0}+\cos \theta}{\cos \theta_{0} \sin ^{2} \theta}\right)^{1 / 2}, 8
\end{gathered}
$$

describing parabolic motion around a central point mass. The angle $\theta_{0}$ is the angle between the orbital plane and the rotation axis, while $\theta$ is the angle from the rotation axis to the particle. For simplicity, the velocities are only considered for motion nearly in the equatorial plane $\left(\theta_{0} \geqslant 89^{\circ}\right)$, making $v_{\theta} \sim 0$. Note that the term $\cos \theta / \cos \theta_{0}$, appearing in both $v_{r}$ and $v_{\phi}$, will go to zero as $\theta_{0} \rightarrow \theta$. Furthermore, most envelopes in this study are rather filamentary and we will be comparing with PV diagrams taken from equatorial regions, making $v_{\theta}$ contributions negligible. A fiducial central object mass of $0.5 M_{\odot}$ is assumed in these models, intended to be a typical protostellar mass in the absence of any real constraints. However, the envelope masses within 10,000 AU are $\sim 1 M_{\odot}$ for the objects that will be considered in this work (Table 1); this is not negligible with respect to the assumed central mass, and we will discuss its possible effects on the kinematics in Section 4.

Other than mass, the only free parameter of the CMU velocity field is the initial angular velocity of the material falling in at the current time. Material falling in from an initial direction given by the angle $\theta_{0}$ from the rotation axis lands on the (flat) disk at a radius $r$, given by

$$
\frac{r}{R_{C}}=\frac{\sin ^{2} \theta_{0}}{1-\cos \theta / \cos \theta_{0}},
$$

where $R_{C}$ is the centrifugal radius,

$$
R_{C}=\frac{R_{0}^{4} \Omega^{2}}{G M} .
$$

Here, $R_{0}$ is the radius that the material fell in from, with angular velocity $\Omega$. The $\cos \theta / \cos \theta_{0}$ terms in Equations (1)- (3) can

\footnotetext{
8 Ulrich (1976) and Chevalier (1983) both have $\sin \theta$ in the denominator, rather than $\sin ^{2} \theta$ as written in Terebey et al. (1984) and Hartmann (2009). The correct term is $\sin ^{2} \theta$; this can be verified by ensuring that the squares of the three velocity components add to $2 G M / R$.
}

then be rewritten in terms of $r / R_{C}$, relating the CMU rotation velocity $\left(v_{\phi}\right)$ to the initial angular velocity $(\Omega)$ of the infalling shell.

To calculate the velocities that would be observed in a PV plot across the equatorial plane, the velocities in the $r, \phi$, and $\theta$ directions must be projected along a line of sight. For simplicity, the line of sight is defined to be the $y$-axis at $z=0$, which makes

$$
v_{\mathrm{los}}=v_{r}\left(\sin i \sin \Phi_{0}\right)+v_{\theta}\left(\cos i \sin \Phi_{0}\right)+v_{\phi}\left(\cos \Phi_{0}\right),
$$

where $\Phi_{0}$ is the projection angle of the envelope within the line of sight, measured counterclockwise from the $x$-axis. $\Phi_{0}$ is unrelated to $\phi$, which is the azimuthal angle of infalling material, and $\Phi_{0}$ only affects the observed velocity distribution in a non-axisymmetric system. The angle $i$ is the inclination of the rotation axis of the system with respect to the plane of the sky; this adds an additional projection term to the observed velocity distribution. However, we only consider velocities calculated at inclination of $90^{\circ}$, meaning that the rotation axis of the model would be in the plane of the sky. This simplification is made to limit free parameters and because most of the observed systems in Paper II have inclinations of $i \geqslant 60^{\circ}$, making this projection effect minimal.

We neglect radiative transfer and chemistry for this initial exploration; this is reasonable because we will compare the models with optically thin molecular line kinematics that trace cold $(T \sim 10 \mathrm{~K})$ molecular gas at the densities expected for protostellar envelopes $\left(n>10^{5} \mathrm{~cm}^{-3}\right.$, i.e., $\mathrm{NH}_{3}$ and $\left.\mathrm{N}_{2} \mathrm{H}^{+}\right)$. These molecules are present with detectable emission from large scales $(\sim 0.1 \mathrm{pc})$ down to radii as small as $\sim 1000 \mathrm{AU}$ (Caselli et al. 2002; Benson \& Myers 1989; Lee et al. 2004; Paper II); however, the abundances of these molecules are not constant with radius. Our models use the simplest possible non-axisymmetric envelope structure to describe the highdensity material and optically thin emission-line kinematics: an $m=2$ single filament. The use of optically thick lines to probe the envelope kinematics would need to consider lower density surrounding material that could contain even higherorder structure resulting in more complex line profiles. Comparison with the numerical models of Smith et al. (2011) with radiative transfer will be the subject of a future paper.

\subsection{Predicted Kinematic Structure}

The left panel of Figure 2 shows the infall streamlines on large scales for the axisymmetric case, and the middle panels show a zoom-in on the inner 1000 AU. The infall streamlines are computed every $15^{\circ}$ in the $\phi$ direction to sample the entire envelope. The projected velocities versus $x$-axis position are used to construct simple PV plots from the individual envelope streamlines, shown in the right panel of Figure 2. Note that we have assumed that the envelope is infalling from 10,000 $\mathrm{AU}$, with no static outer core. However, we know that there is substantial mass on larger scales for all these objects measured via $8 \mu \mathrm{m}$ extinction, continuing to increase from $0.05 \mathrm{pc}$ to $0.15 \mathrm{pc}$ in radius (Paper I). The masses compared with the observed linewidths indicate that the envelopes are gravitationally bound (Paper II); therefore, we expect that material from larger scales will be falling in, but under the influence of greater enclosed mass. Thus, the largescale velocities from the CMU velocity field are lower limits.

To approximate a filamentary envelope in the same manner as the axisymmetric envelope, we only calculate three streamlines on either side of the protostar, spanning $\Delta \phi=30^{\circ}$ in azimuth at 



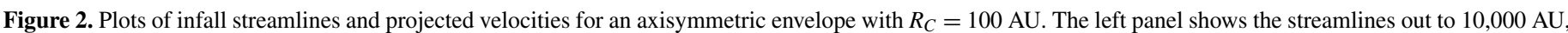

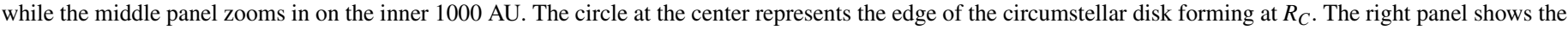
predicted PV structure for the axisymmetric envelope.

scales larger than $R_{C}$. The geometry of the infall streamlines is shown in the top left and middle panels of Figure 3. The PV plots are then constructed in the same manner as the axisymmetric case and shown in the right panels of Figure 3. We have assumed that matter is infalling from $10,000 \mathrm{AU}$, the same as the axisymmetric case. Note that the region of velocity space enclosed by the filament model is smaller than the axisymmetric case; the velocity streamlines plotted in the filament model represent a subset of those plotted for the axisymmetric model. Then the effects of viewing the filamentary envelope at $\Phi_{0}=$ $\pm 30^{\circ}$ projection angles are shown in the middle and bottom rows of Figure 3. $\Phi_{0}$ specifically refers to the projection angle of the middle streamline on either side of the envelope in Figure 3. Variations of $\Phi_{0}$ in non-axisymmetric systems can clearly introduce apparent velocity shifts and gradients that are not present in axisymmetric geometries.

Substantially different velocity structures are evident in the filamentary PV plots, especially when viewed with different projection angles within the plane of the sky, as compared with axisymmetric collapse in Figure 2. Notably the velocity shift across the filamentary envelopes on scales $>1000 \mathrm{AU}$ is not due to rotation velocity; rather, it is due to the infall velocity being projected along the line of sight. The schematic plots of envelope kinematics in Figure 1 and the PV plots derived from the CMU model for axisymmetric and filamentary envelopes can be used as benchmarks to compare with our observational data. This will potentially enable us to distinguish between axisymmetric and non-axisymmetric infalling envelopes, as well as velocity gradients that are due to rotation or projected infall.

\section{OBSERVATIONAL AND MODEL RESULTS}

We have selected five protostellar systems from Paper II to compare with the kinematic models, all having relatively uncomplicated morphological structure and/or ordered kinematic structure. These features are important because the interpretation of the velocity structures is complicated by the often complex envelope structures (Papers I and II). We examine channel maps and PV diagrams for each protostellar system to better understand the dynamical processes behind the observed kinematic structure. We compare the models and data using PV plots of the simplistic axisymmetric or filamentary collapse models described in the previous section. The final model parameters
Table 2

Model Parameters

\begin{tabular}{lccrc}
\hline \hline Source & Model & $\begin{array}{c}M_{\text {cent }} \\
\left(M_{\odot}\right)\end{array}$ & $\begin{array}{c}R_{C} \\
(\mathrm{AU})\end{array}$ & $\begin{array}{c}\text { Proj. Angle } \\
\left({ }^{\circ}\right)\end{array}$ \\
\hline L1157 & Filament & 0.5 & 100 & 15 \\
L1165 & Filament & 0.5 & 10 & 15 \\
CB 230 & Filament & 0.5 & 10 & 20 \\
IRAS 16253-2429 & Filament/Axisymmetric & 0.1 & 100 & 0 \\
HH 211 & Filament & 0.5 & 10 & 15 \\
\hline
\end{tabular}

Note. Parameters of models that describe the PV data.

are listed in Table 2. These models do not provide unique fits, given the geometric uncertainties, but they illustrate the basic features and issues involved in determining the processes giving rise to envelope kinematics. Also note that we have not combined zero-spacing data with our interferometric data resulting in the large-scale emission being filtered out and possibly influencing our kinematic data. However, we show in Paper II that the velocity fields observed in the single-dish and interferometric data are consistent at large scales, indicating that the spatial filtering by the interferometer is not biasing our kinematic results.

\section{1. $L 1157$}

L1157 is located in Cepheus at a distance of $\sim 300 \mathrm{pc}$ and is an example of a rather well ordered, highly flattened envelope. The flattened envelope is observed in $8 \mu \mathrm{m}$ extinction, as well as $\mathrm{N}_{2} \mathrm{H}^{+}$and $\mathrm{NH}_{3}$ emission. The $\mathrm{N}_{2} \mathrm{H}^{+}$integrated intensity map is shown in the upper left panel of Figure 4 . The envelope mass was found to be at least $\sim 0.86 M_{\odot}$ from $8 \mu \mathrm{m}$ extinction (Paper I) and possibly an additional $\sim 0.7 M_{\odot}$ inferred from the $\mathrm{N}_{2} \mathrm{H}^{+}$ emission (Paper II). We have further shown in Paper I that the flattening reflects a filamentary structure in three dimensions rather than a sheet extended within the plane of the sky. The scale heights of the short envelope axis were $\sim 1600$ AU across all radii (except the inner $10^{\prime \prime}$ ), consistent with a hydrostatic filament; a sheet would be a factor of three more extended in the vertical direction. Thus, L1157 may be well described by an axisymmetric filament, one of the ideal cases shown in Figure 1.

The channel maps in Figure 4 show several kinematic features in this system. In the top and bottom rows, there is 

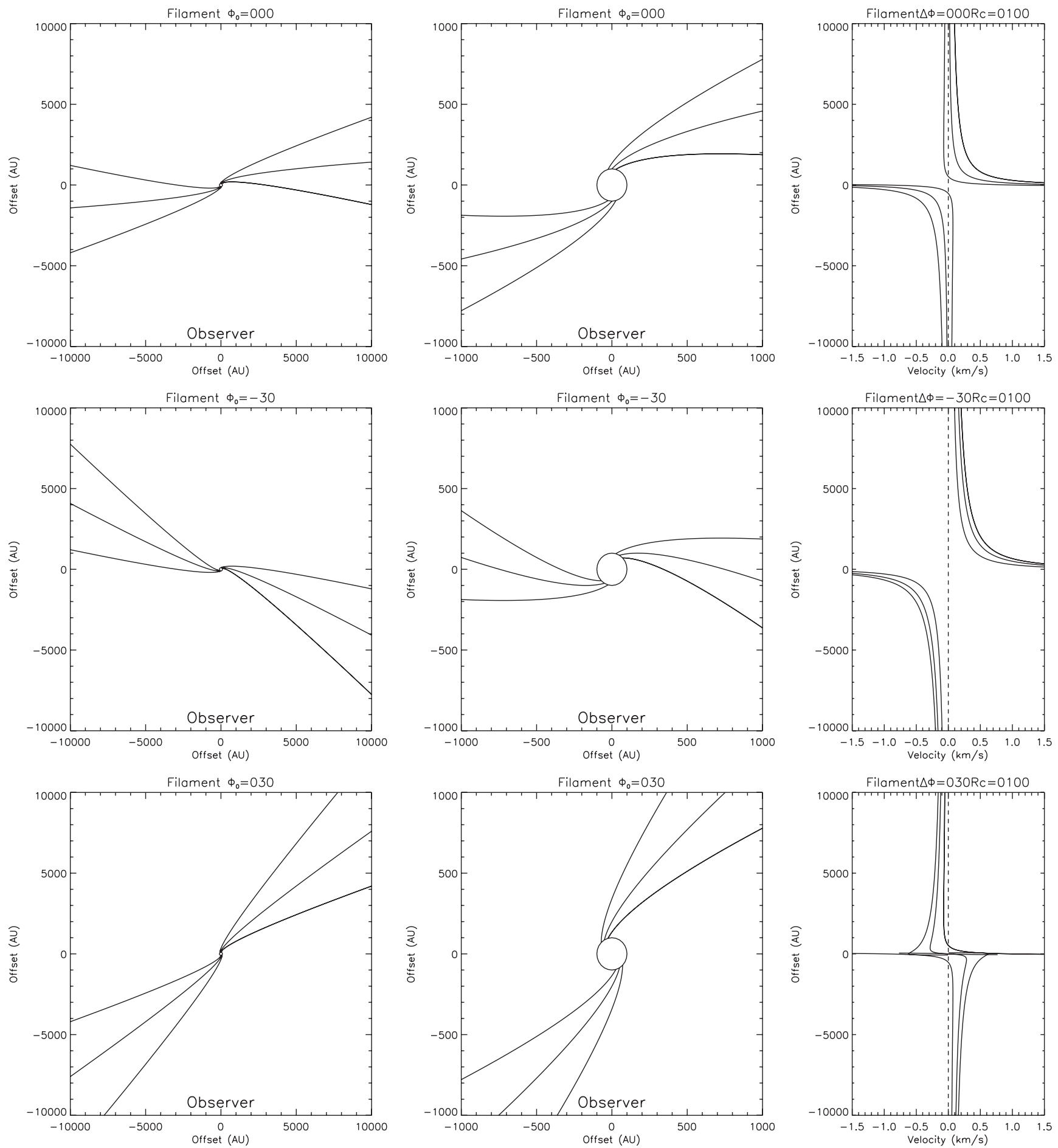

Figure 3. Plots of infall streamlines for a filamentary envelope with $R_{C}=100 \mathrm{AU}$. From top to bottom, filamentary envelopes are shown with projection angles of $\Phi_{0}=0^{\circ},-30^{\circ}$, and $30^{\circ}$. The left panel shows the streamlines out to $10,000 \mathrm{AU}$, while the middle panel zooms in on the inner $1000 \mathrm{AU}$. The circle at the center represents the edge of the circumstellar disk forming at $R_{C}$. The right panel shows the PV structure of the filamentary streamlines. The filamentary envelopes at a particular rotation angle can be thought of as showing a selected portion of the axisymmetric PV diagram.

higher velocity $\mathrm{N}_{2} \mathrm{H}^{+}$emission north and south of the protostar extended along the outflow. This emission was attributed to outflow interactions in Paper II. In the middle panels, there are emission peaks from the inner envelope on $\pm 5^{\prime \prime}$ scales present in all the channels. However, on scales $>77^{\prime \prime}$ we see that the east side of the envelope comes into view at blueshifted velocities and the west side at redshifted velocities. This traces a clear large-scale velocity gradient that may be attributed to rotation or projected infall. Note that the extension of $\mathrm{N}_{2} \mathrm{H}^{+}$emission southeast of the protostar traces emission from the outflow cavity wall and also appears to reflect outflow entrainment.

The PV plot in Figure 5 also shows high-velocity emission due to the outflow-envelope interaction extending toward redshifted velocities on both sides of the envelope, coincident with the $\mathrm{N}_{2} \mathrm{H}^{+}$emission peaks. Thus, any indication of high-velocity emission due to rotation or infall at small scales is masked by the outflow effects. However, Gueth et al. (1997) appear to have detected a smaller scale velocity gradient in $\mathrm{C}^{18} \mathrm{O}$ emission. 


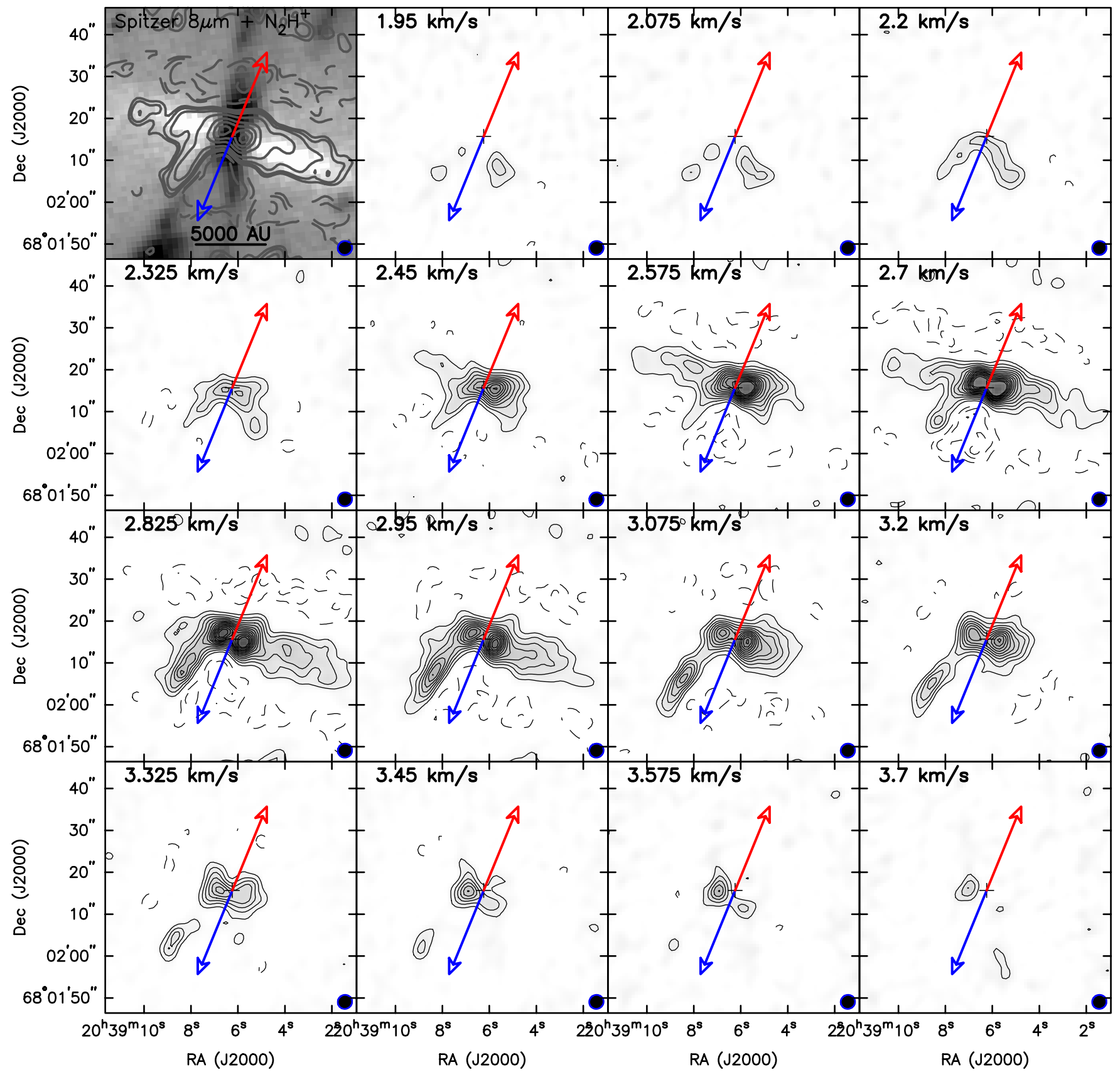

Figure 4. L1157: channel maps of the isolated $\mathrm{N}_{2} \mathrm{H}^{+}\left(J F_{1} F=101 \rightarrow 012\right)$ line; the blue and red arrows mark the outflow direction, and the cross marks the location of the protostar. The higher velocity data in the top and bottom rows mainly trace the likely outflow-entrained material, while the middle two rows primarily reflect emission due to envelope kinematics. Note the velocity gradient of the large-scale material from $2.45 \mathrm{~km} \mathrm{~s}^{-1}$ to $3.075 \mathrm{~km} \mathrm{~s}^{-1}$. The large-scale emission on the east and west sides of the envelope has a rather constant velocity. The extension of $\mathrm{N}_{2} \mathrm{H}^{+}$emission to the south traces an outflow cavity wall whose kinematics also reflect some outflow entrainment. Contours in the channel maps start at $\pm 3 \sigma$ and increase by $\pm 3 \sigma$ intervals, where $\sigma=0.08 \mathrm{~K}$. The contours in the integrated intensity map are $\pm 6 \sigma, 9 \sigma, 15 \sigma, 30 \sigma, 60 \sigma, \ldots$, where $\sigma=0.02 \mathrm{~K} \mathrm{~km} \mathrm{~s}^{-1}$.

(A color version of this figure is available in the online journal.)

On scales $>7^{\prime \prime}$ in Figure 5, the envelope velocities are nearly constant with a systematic $\sim 0.1 \mathrm{~km} \mathrm{~s}^{-1}$ velocity shift between the east and west sides of the envelope, as seen in the channel maps. The larger scale features, with their nearly constant velocity, are quite similar to the filament model shown in the bottom right panel of Figure 3. At the same time, the velocity structure is dissimilar to uniform rotation and the axisymmetric infall model (cf. Chiang et al. 2010). A filament model has been overlaid on the data in the PV plot in Figure 5, showing that the fit is reasonable, except in the inner envelope where the kinematic structure is not accurately probed. The model is projected within the plane of the sky by $\Phi_{0}=15^{\circ}$ and has a centrifugal radius of $100 \mathrm{AU}$ (Table 2).

\section{2. $L 1165$}

L1165 is also located in the Cepheus region, and the protostar is forming within a larger scale filamentary structure (Paper I). The $\mathrm{N}_{2} \mathrm{H}^{+}$emission on small scales, shown in the upper left panel of Figure 6, traces a structure extended normal to the outflow, peaking just southeast of the protostar. A lower limit on the envelope mass is measured to be $\sim 1.1 M_{\odot}$ from 



Figure 5. L1157: the left panel shows the IRAC $8 \mu \mathrm{m}$ image with PdBI $\mathrm{N}_{2} \mathrm{H}^{+}$integrated intensity contours overlaid of the isolated $\mathrm{N}_{2} \mathrm{H}^{+}$line $\left(J F_{1} F=101 \rightarrow 012\right)$. The dashed lines mark the regions where the position-velocity cut was taken and point to respective ends of the PV plot in the right panel. The position of the protostar/continuum source is marked with a white cross. The position-velocity cut is shown for the isolated $\mathrm{N}_{2} \mathrm{H}^{+}$line, showing that the two lumps of $\mathrm{N}_{2} \mathrm{H}^{+}$ emission are directly associated with regions of increased linewidth, likely due to outflow effects. Outside of this region, the linewidth becomes fairly narrow in the outer envelope with constant velocity. The green lines overlaid are a filamentary collapse model with $R_{C}=100 \mathrm{AU}$ and a projection of $\Phi_{0}=15^{\circ}$. The contours in the integrated intensity map are $\pm 6 \sigma, 9 \sigma, 15 \sigma, 30 \sigma, 60 \sigma, \ldots$, where $\sigma=0.02 \mathrm{~K} \mathrm{~km} \mathrm{~s}^{-1}$; the contours in the PV diagram are $\pm 3 \sigma$ and in increments of $3 \sigma$ where $\sigma=0.17 \mathrm{~K}$.

(A color version of this figure is available in the online journal.)

$8 \mu \mathrm{m}$ extinction in Paper I, with perhaps at least an additional $\sim 0.4 M_{\odot}$ inferred from $\mathrm{N}_{2} \mathrm{H}^{+}$emission in Paper II. The $\mathrm{N}_{2} \mathrm{H}^{+}$ channel maps for L1165 are shown in Figure 6, indicating a clear velocity gradient across the protostar (normal to the outflow direction). The velocity on the northwest side of the protostar is blueshifted and nearly constant. The emission then becomes redshifted on the southeast side of the envelope, with the highest velocity emission being redshifted, near the protostar. There is evidence of some higher velocity blueshifted emission adjacent to the protostar on the northwest side in the channel maps, but it is not as definitive as the redshifted emission.

The PV diagram in Figure 7 shows that the $\mathrm{N}_{2} \mathrm{H}^{+}$emission northwest of the protostar has a very narrow linewidth and a roughly constant velocity; the broadest linewidth is southeast of the protostar, near the $\mathrm{N}_{2} \mathrm{H}^{+}$peak. Southeast of the peak linewidth, the line rapidly becomes narrow again, while becoming more blueshifted. The channel maps show that the $\mathrm{N}_{2} \mathrm{H}^{+}$ kinematics are unlikely to be outflow related, owing to their location orthogonal to the outflow. The PV structure in Figure 7 is most similar to the filamentary infall model; its velocities are overlaid in Figure 7. The model is able to approximate the observed velocity structure, though there are discrepancies; for L1165 the model is projected by $\Phi_{0}=15^{\circ}$ within the plane of the sky and has a centrifugal radius of $10 \mathrm{AU}$ (Table 2). The small centrifugal radius of the model implies that the data are consistent with little rotation on scales probed by $\mathrm{N}_{2} \mathrm{H}^{+}$.

As Figures 6 and 7 show, $\mathrm{N}_{2} \mathrm{H}^{+}$is only able to trace emission to within $R \sim 1200 \mathrm{AU}$ of the protostar. A special feature found in $\mathrm{L} 1165$ is that $\mathrm{HCO}^{+}(J=1 \rightarrow 0)$ emission is able to trace small-scale kinematics with higher velocity in the inner envelope, shown in Figure 8. The redshifted and blueshifted components are extended normal to the outflow at radii of $3^{\prime \prime}$ (600 AU) from the protostar. This emission lies inside the broadest $\mathrm{N}_{2} \mathrm{H}^{+}$emission, suggesting that it traces smaller scale kinematic structure, in agreement with the small-scale model velocities that are overlaid in Figure 8. Furthermore, the location of the blueshifted and redshifted high-velocity emission on opposing sides of the envelope with opposite velocities, along with the small velocity width at large scales in the $\mathrm{N}_{2} \mathrm{H}^{+}$ emission, is consistent with what is expected for a filamentary envelope (Figure 3). A similar high-velocity $\mathrm{HCO}^{+}$feature was found in RNO43 (Paper II).

\section{3. $C B 230$}

CB 230 was classified as a "one-sided" envelope in Paper I, given its strongly asymmetric distribution of $8 \mu \mathrm{m}$ extinction; this was reflected on large scales in the single-dish $\mathrm{N}_{2} \mathrm{H}^{+}$ map shown in Paper II. We measure a lower limit on the envelope mass within $0.05 \mathrm{pc}$ of $\sim 1.1 M_{\odot}$ in Paper I from $8 \mu \mathrm{m}$ extinction, but there is clearly mass on small scales that we do not probe. The smaller scale emission probed by $\mathrm{NH}_{3}$, shown in the upper left panel of Figure 9, is slightly asymmetric, but well ordered as a whole. The mass inferred from the small-scale $\mathrm{NH}_{3}$ emission in Paper II was $\sim 4.8 M_{\odot}$, but highly uncertain. There is also a depression in the $\mathrm{NH}_{3}$ emission in the inner envelope, coincident with the protostar. This effect is attributed to destruction of $\mathrm{NH}_{3}$ in the inner envelope (Paper II). The velocity structure of the $\mathrm{NH}_{3}$ emission is shown by channel maps in Figure 9. Because the main $\mathrm{NH}_{3}(1,1)$ lines are a pair separated by $0.2 \mathrm{~km} \mathrm{~s}^{-1}$, the last three panels in the middle row are blends of blueshifted and redshifted emission, while the rest of the plots reflect mostly unblended emission. The channel maps show that there is a clear, well-ordered velocity gradient across the envelope from east to west, normal to the outflow.

The PV diagram in Figure 10, in contrast to the channel maps, is derived from $\mathrm{NH}_{3}$ satellite emission lines that are separated 


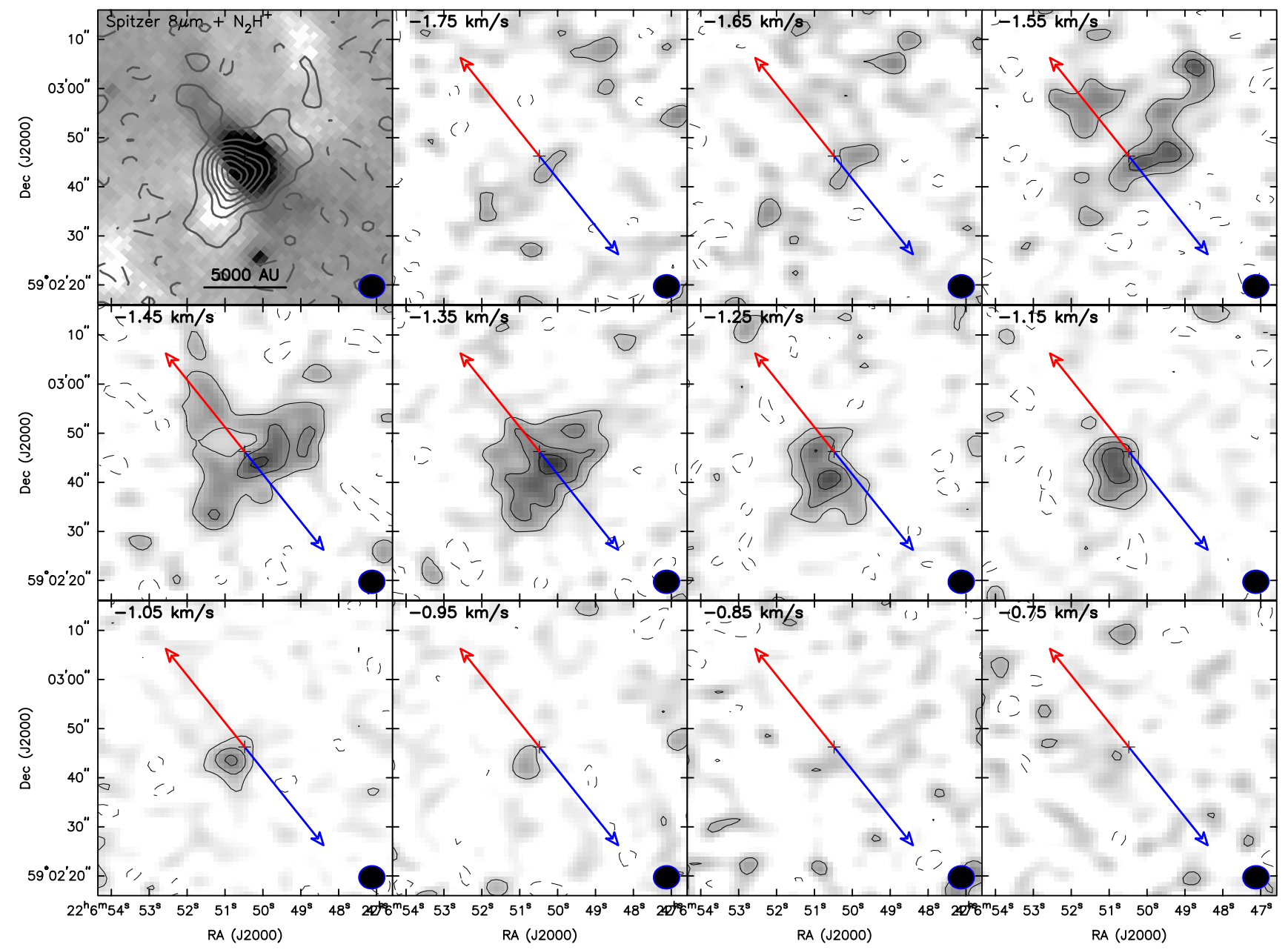

Figure 6. L1165: channel maps of the isolated $\mathrm{N}_{2} \mathrm{H}^{+}\left(J F_{1} F=122 \rightarrow 011\right)$ line; the blue and red arrows mark the outflow direction, and the cross marks the location of the protostar. There is a rather well behaved global velocity structure in L1165 from blueshifted northwest of the protostar and redshifted to the southeast. There does not appear to be significant outflow effects on the kinematic structure. There is also higher velocity redshifted emission apparent on small scales near the protostar, but very little blueshifted emission with higher velocities. The contours in the integrated intensity map are $\pm 2.5 \sigma, 3 \sigma, 6 \sigma, \ldots$, where $\sigma=0.207 \mathrm{~K} \mathrm{~km} \mathrm{~s}{ }^{-1}$; the contours in the channel maps are $\pm 3 \sigma, 6 \sigma, \ldots$, where $\sigma=0.4 \mathrm{~K}$.

(A color version of this figure is available in the online journal.)

by $\sim 0.4 \mathrm{~km} \mathrm{~s}^{-1}$. This makes the emission appear more distinct than if the $\mathrm{PV}$ diagram were plotted using the main $\mathrm{NH}_{3}$ lines. The PV plot and channel maps show that the emission on the east and west sides of the envelope has a rather constant velocity and there is an abrupt shift from blueshifted to redshifted emission starting at the location of the protostar.

While a measure of the small-scale velocities is missing, the emission on scales $>1000 \mathrm{AU}$ is most consistent with the infalling filament, owing to the constant velocity emission out to large scales and the relatively narrow linewidth. The constant velocity emission is dissimilar from what would be expected for uniform rotation, which would have linearly increasing velocity out to large scales. The filament model is overlaid on the data in Figure 10, having projected by $\Phi_{0}=20^{\circ}$, and has $R_{C}=10 \mathrm{AU}$. The model does not exactly describe the data, but substantial mass in the inner envelope implied by the $\mathrm{NH}_{3}$ data could make the velocity shift slightly larger on the scales probed.

\subsection{IRAS 16253-2429}

IRAS $16253-2429$ is the most nearby object in our sample, located in Ophiuchus $(d \sim 125 \mathrm{pc})$, and one of the most symmetric, spherical envelopes in $8 \mu \mathrm{m}$ extinction and $\mathrm{N}_{2} \mathrm{H}^{+}$ emission, plotted in Figure 11. The velocity gradient in this envelope is quite small, illustrated by the channel maps with the eastern part of the envelope coming into view just one channel before the rest. Figure 12 shows the PV diagram; the emission has a roughly constant linewidth and velocity across the envelope and only a slight velocity gradient is evident. Furthermore, the $\mathrm{N}_{2} \mathrm{H}^{+}$emission is depressed toward the protostar, as compared with the surrounding emission. This effect appears to be a depletion/destruction process since the lines are not optically thick (Paper II).

This is the only envelope in our sample that can be reasonably described by an axisymmetric envelope model; however, it was necessary to lower the mass to $0.1 M_{\odot}$, such that the linewidth from infall was not too large. The lower mass is appropriate for this source given its low luminosity $\left(L_{\mathrm{bol}}=\right.$ $0.25 L_{\odot}$; the envelope does have at least $0.8 M_{\odot}$ of material within $10,000 \mathrm{AU}$, with increasing mass on yet larger scales, suggesting that the central object could be more massive). On the scales probed by the CARMA data $(R \sim 3125 \mathrm{AU}$; $\left.25^{\prime \prime}\right)$ the lower limit on the mass measured from the $8 \mu \mathrm{m}$ extinction data is just $0.2 M_{\odot}$. Between $3000 \mathrm{AU}$ and $6000 \mathrm{AU}$ 


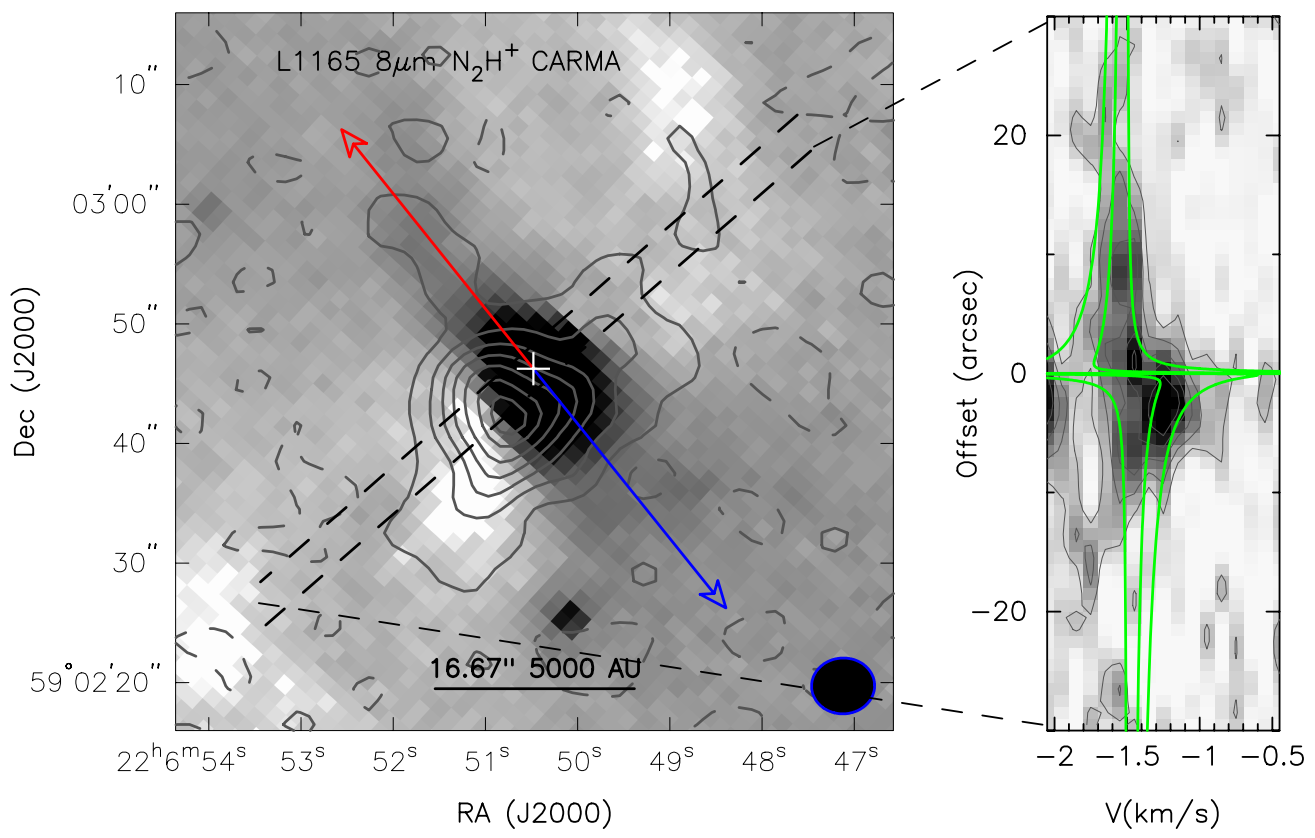

Figure 7. L1165: the left panel shows the IRAC $8 \mu \mathrm{m}$ image with CARMA $\mathrm{N}_{2} \mathrm{H}^{+}$integrated intensity contours overlaid. The dashed lines mark the regions where the PV cut was taken and point to respective ends of the PV plot in the right panel showing the $\mathrm{N}_{2} \mathrm{H}^{+}\left(J F_{1} F=122 \rightarrow 011\right)$ transition. The position of the protostar/continuum source is marked with a white cross. Northwest of the protostar the lines tends to be more narrow than on the southeast side of the protostar, where the $\mathrm{N}_{2} \mathrm{H}^{+}$emission is both broadshifted and redshifted. The green lines overlaid are a filamentary collapse model with $R_{C}=10 \mathrm{AU}$ and a projection of $\Phi_{0}=15^{\circ}$. The contours in the integrated intensity map are $\pm 2.5 \sigma, 3 \sigma, 6 \sigma, \ldots$, where $\sigma=0.207 \mathrm{~K} \mathrm{~km} \mathrm{~s}^{-1}$; the contours in the PV diagram are $\pm 3 \sigma$, increasing in increments of $3 \sigma$, where $\sigma=0.225 \mathrm{~K}$

(A color version of this figure is available in the online journal.)

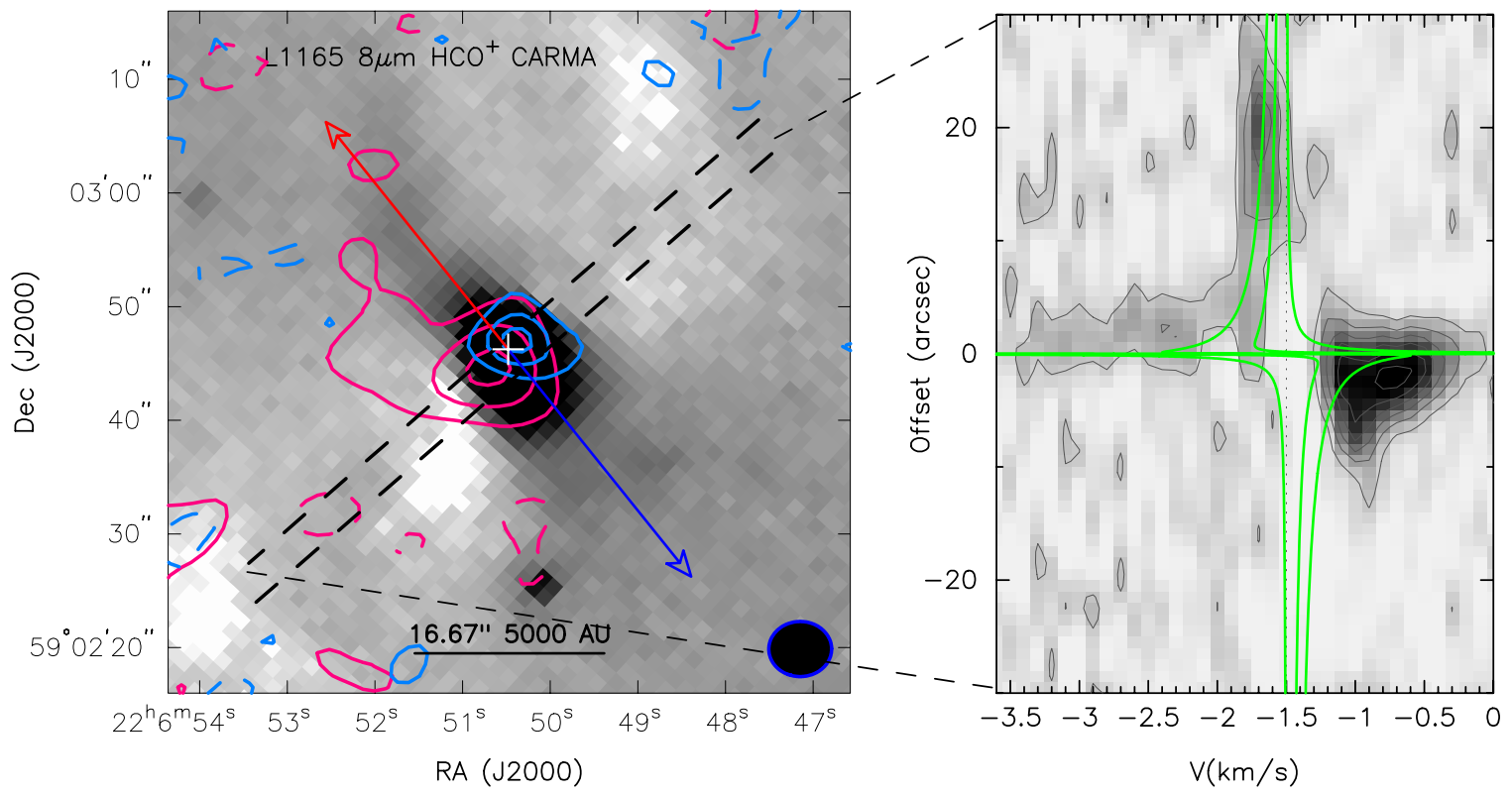

Figure 8. L1165: the left panel shows the IRAC $8 \mu \mathrm{m}$ image with CARMA $\mathrm{HCO}^{+}(J=1 \rightarrow 0)$ blueshifted and redshifted emission, summed over -3.5 to $-2 \mathrm{~km} \mathrm{~s}^{-1}$ and -1.0 to $0 \mathrm{~km} \mathrm{~s}^{-1}$, plotted as blue and red contours, respectively. The contour levels are $3 \sigma, 6 \sigma$, and $8.25 \sigma(\sigma=0.175 \mathrm{~K})$ for the blueshifted emission and $3 \sigma$, $9 \sigma$, and $18 \sigma(\sigma=0.212 \mathrm{~K})$ for the redshifted emission. The blueshifted and redshifted emission from $\mathrm{HCO}^{+}$is located symmetrically about the protostar, normal to the outflow. The dashed lines mark the regions where the PV cut was taken and point to respective ends of the PV plot in the right panel. The position of the protostar/continuum source is marked with a white cross. The PV cut shows that the blueshifted and redshifted emission traces higher velocity material and there is a slight gradient of material going to higher velocity closer to the continuum source. The line-center velocity of $-1.5 \mathrm{~km} \mathrm{~s}^{-1}$ is plotted as the dotted black line. The green lines overlaid are a filamentary collapse model with $R_{C}=10 \mathrm{AU}$ and a projection of $\Phi_{0}=15^{\circ}$. The PV plot contours start at $3 \sigma$ and increase in $3 \sigma$ intervals $(\sigma=0.2)$. (A color version of this figure is available in the online journal.)

scales, probed mainly by the single-dish data from Paper II, the linewidth from spherical infall with dominant envelope mass would be $\sim 0.2 \mathrm{~km} \mathrm{~s}^{-1}$. Thus, the data for this source appear to be consistent with spherical infall given the low mass of the envelope; however, solid-body rotation and filamentary collapse cannot be ruled out by these data. The lack of a significant velocity structure makes the kinematic models degenerate for this object. 


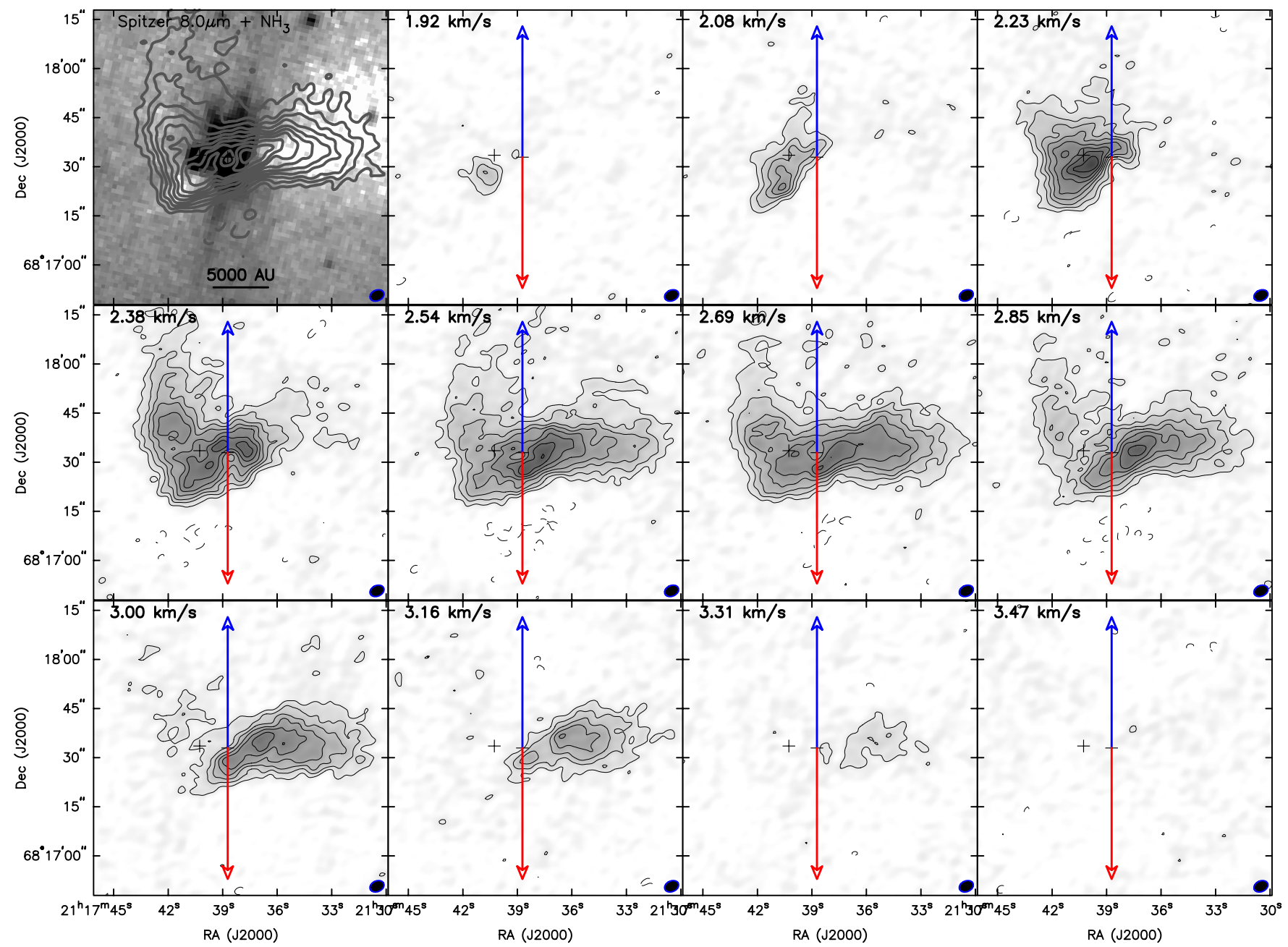

Figure 9. $\mathrm{CB} 230: \mathrm{NH}_{3}$ integrated intensity map of the main $\mathrm{NH}_{3}(1,1)$ lines (upper left), and channel maps of the two main $\mathrm{NH}_{3}(1,1)$ lines are shown in the rest of the panels. The protostars are marked with crosses, and the outflow direction is drawn from the main protostar. The emission between 2.54 and $2.85 \mathrm{~km} \mathrm{~s}^{-1}$ consists of blended emission from both lines, while the remaining emission is mostly free of blending. The channel maps show a clear velocity gradient across the source, appearing normal to the outflow. Note how the emission tends to avoid the location of the main protostar, owing to destruction of $\mathrm{NH}_{3}$ near the protostar. The contours in the integrated intensity map start at $\pm 3 \sigma$, increasing in increments of $3 \sigma$ and $\sigma=0.195 \mathrm{~K} \mathrm{~km} \mathrm{~s}^{-1}$; the contours in the integrated intensity map are $\pm 3 \sigma, 6 \sigma, \ldots$, where $\sigma=0.325 \mathrm{~K}$.

(A color version of this figure is available in the online journal.)

\section{5. $\mathrm{HH} 211$}

HH 211 was classified as an irregular envelope in Paper I; however, its structure in $\mathrm{N}_{2} \mathrm{H}^{+}$appears filamentary, but not as regular as $\mathrm{L} 1157$ or CB 230 (Figure 13). The peak $\mathrm{N}_{2} \mathrm{H}^{+}$ emission is just southwest of the protostar, coincident with the $\mathrm{NH}_{3}$ peak (Tanner \& Arce 2011). The mass of this envelope is found to be $\sim 1.1 M_{\odot}$ from $8 \mu \mathrm{m}$ extinction (Paper I) and $\sim 0.65 M_{\odot}$ in the inner envelope from $\mathrm{N}_{2} \mathrm{H}^{+}$emission (Paper II). We also find a velocity gradient that is approximately normal to the outflow and changing. The PV plot in Figure 14 shows that to the northeast, the velocity gradient is smaller, while to the southwest it increases, where it blends with an apparent second velocity component (Paper II and Tanner \& Arce 2011).

On the whole, the data appear most consistent with solid-body rotation, but this is uncertain given apparent velocity gradient change and the irregularity of the envelope. The smaller gradient to the northeast is consistent with the infalling filament models, which we overlay in Figure 14. The model is projected by $\Phi_{0}=15^{\circ}$ and has $R_{C}=10 \mathrm{AU}$. The apparent change of the velocity gradient could be due to complex projection effects or the surrounding environment. Even though there is substantial mass measured in this envelope with an asymmetric distribution, it is not plausible that it could generate such a large linear velocity gradient from infall alone in the context of these simple models. Furthermore, the linewidth of the envelope northeast of the protostar is substantially more narrow than the linewidth southwest of the protostar. At the $\mathrm{N}_{2} \mathrm{H}^{+}$peak, the linewidth broadens substantially and could be related to the outflow as suggested in Tanner \& Arce (2011), rather than inner envelope kinematics.

\section{DISCUSSION}

The direction of the jet and outflow is thought to correspond to the rotation axis of the protostar and disk (e.g., Shu et al. 1987). Since circumstellar disks form in the inner envelope owing to conservation of angular momentum, it has been natural to interpret large-scale velocity gradients that are normal to the outflow as rotation (e.g., Goodman et al. 1993; Caselli et al. 2002; Belloche et al. 2002; Belloche \& André 2004; Chen et al. 2007; Paper II). If velocity gradients probe rotation, they give a measure of the angular momentum in a collapsing envelope, 


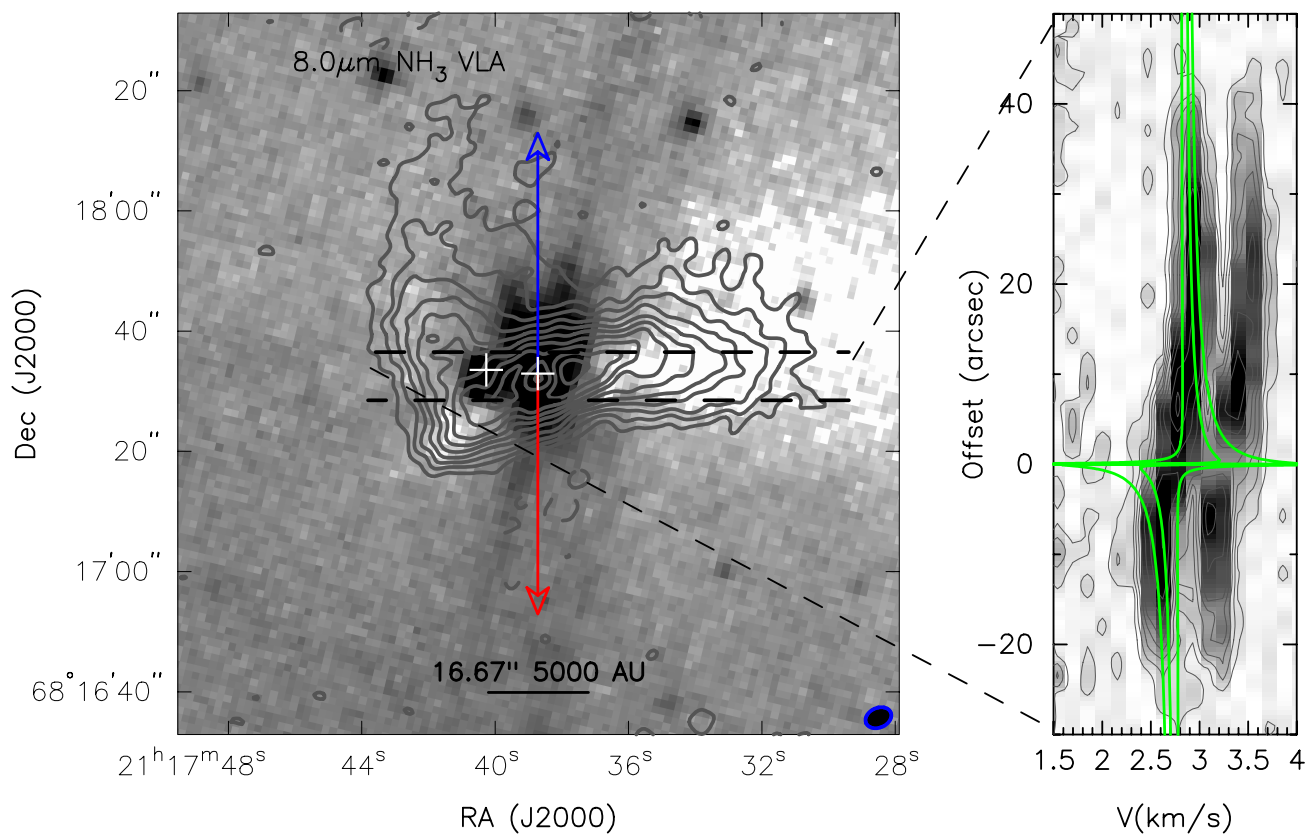

Figure 10. CB 230: same as Figure 5 but with VLA NH$(1,1)$ observations, and the secondary source is also marked with a white cross. The PV diagram in the right panel appears different from those constructed from $\mathrm{N}_{2} \mathrm{H}^{+}$because the $\mathrm{NH}_{3}$ emission consists of two blended hyperfine lines; the $\mathrm{NH}_{3}$ satellite lines with greater separation are shown in the PV plot rather than the more blended main lines. The line-center velocity from the $\mathrm{NH}_{3}$ emission traces an abrupt velocity shift from redshifted to blueshifted emission. The transition region starts at the protostar and does not finish until $+10^{\prime \prime}$. The $\mathrm{NH}_{3}$ linewidth does not show much detail other than having its peak coincident with the highest intensity $\mathrm{NH}_{3}$ emission. The green lines overlaid are a filamentary collapse model with $R_{C}=10 \mathrm{AU}$ and a projection of $\Phi_{0}=20^{\circ}$. The contours in the integrated intensity map start at $\pm 3 \sigma$, increasing in increments of $3 \sigma$, where $\sigma=0.195 \mathrm{~K} \mathrm{~km} \mathrm{~s}^{-1}$; the contours in the PV diagram start at $\pm 3 \sigma$, increasing in increments of $3 \sigma$, where $\sigma=0.11 \mathrm{~K}$.

(A color version of this figure is available in the online journal.)
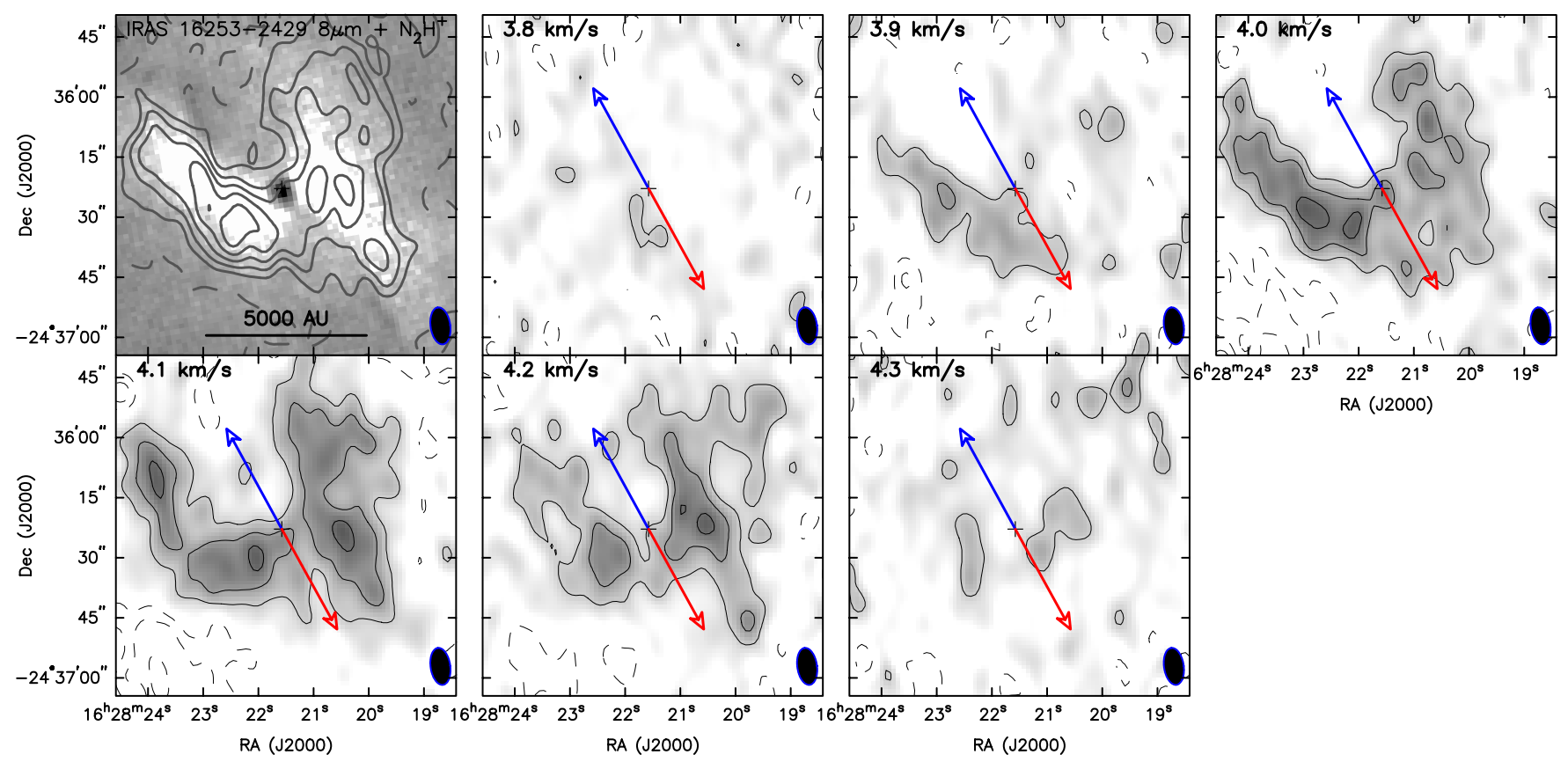

Figure 11. IRAS 16253-2429: channel maps of the isolated $\mathrm{N}_{2} \mathrm{H}^{+}\left(J F_{1} F=122 \rightarrow 011\right)$ line; the blue and red arrows mark the outflow direction and the cross marks the location of the protostar. IRAS $16253-2429$ is the envelope that is most nearly round. The velocity gradient across the envelope is very small but is clearly seen in the channel maps with emission on the eastern side of the envelope coming into view at blueshifted velocities and the western side with redshifted velocities. The green lines overlaid are an axisymmetric collapse model with $R_{C}=100 \mathrm{AU}$ and $M_{C}=0.1 M_{\odot}$. Contours in the channel maps start at $\pm 3 \sigma$, increasing in increments of $\pm 3 \sigma$, where $\sigma=0.08 \mathrm{~K}$. The contours in the integrated intensity map are $\pm 2.5 \sigma, 3 \sigma, 6 \sigma, \ldots$, where $\sigma=0.207 \mathrm{~K} \mathrm{~km} \mathrm{~s}^{-1}$.

(A color version of this figure is available in the online journal.)

enabling the centrifugal radius to be calculated, the radius where a rotationally supported disk will form. However, it was shown in Paper I that envelopes are often asymmetric and filamentary and in Paper II that the large-scale kinematics are often quite complex as compared with a linear velocity gradient. These factors, along with the examples shown in Figure 1, make it uncertain whether or not velocity gradients in these envelopes reflect rotation alone. The qualitative arguments shown in 


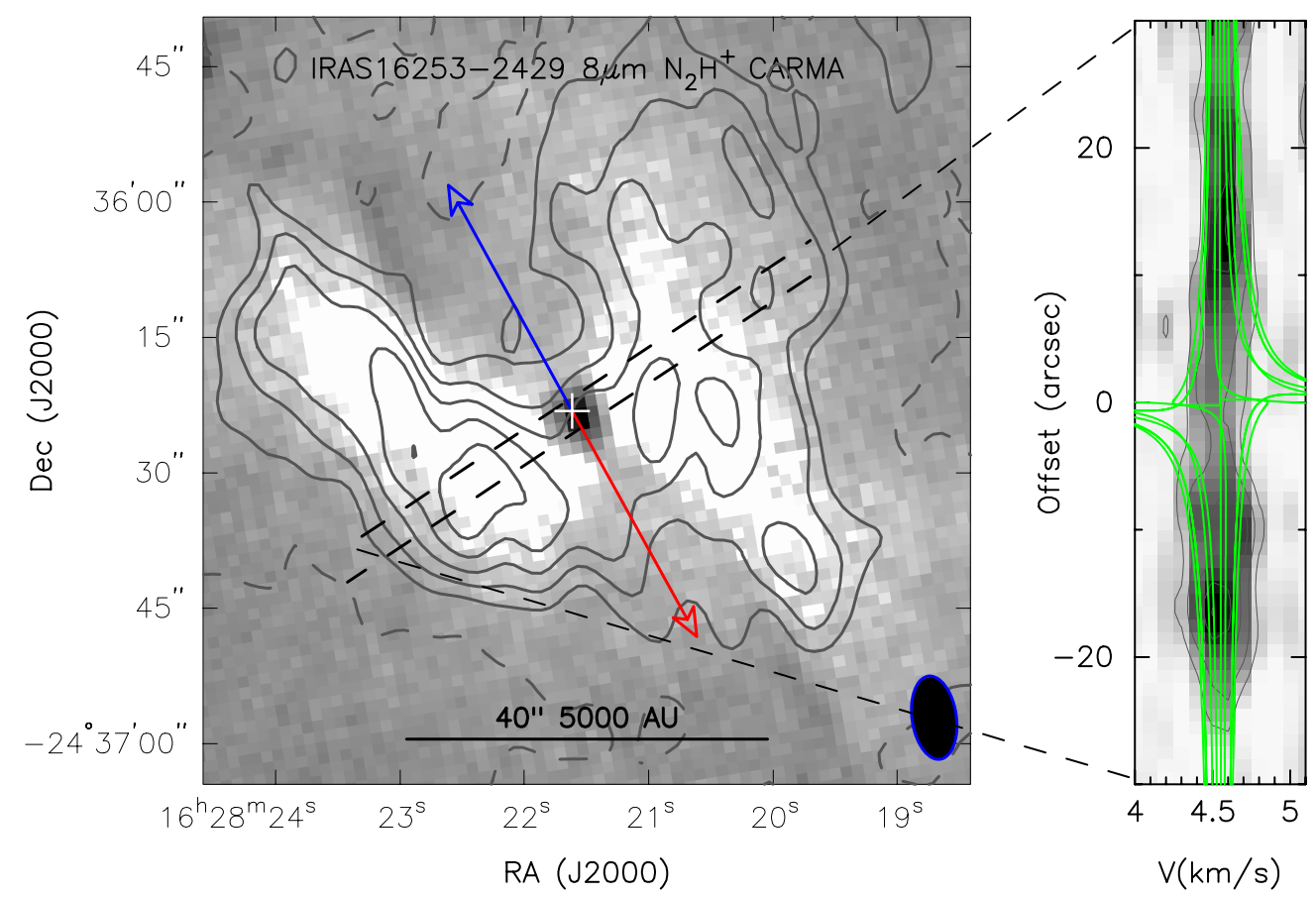

Figure 12. IRAS 16253-2429: same as Figure 5 but with CARMA $\mathrm{N}_{2} \mathrm{H}^{+}$observations of the $\left(J F_{1} F=122 \rightarrow 011\right)$ transition. The $\mathrm{N}_{2} \mathrm{H}^{+}$emission matches up quite well with the $8 \mu \mathrm{m}$ extinction. Furthermore, the $\mathrm{N}_{2} \mathrm{H}^{+}$appears peaked around the protostar and not coincident with it. This is shown as the decreased emission in the $\mathrm{PV}$ plot in the right panel. The velocity gradient in the PV plot is minute but is present. The green lines overlaid are a filamentary collapse model with $R_{C}=10$ AU and a projection of $\Phi_{0}=15^{\circ}$. The contours in the integrated intensity map start at $\pm 3 \sigma$, increasing in increments of $3 \sigma$, where $\sigma=0.22 \mathrm{~K} \mathrm{~km} \mathrm{~s}{ }^{-1}$; the contours in the PV diagram start at $\pm 3 \sigma$, increasing in increments of $3 \sigma$, where $\sigma=0.25 \mathrm{~K}$.

(A color version of this figure is available in the online journal.)

Figure 1 are reinforced by the simple models of axisymmetric and filamentary collapse shown in Figures 2 and 3.

One of the keys to disentangling the kinematics in protostellar envelopes is higher resolution data provided by interferometers. This is because rotation velocities are expected to become most important on small scales owing to conservation of angular momentum. Thus, even if the velocity structure on larger scales probed by single-dish observations is not wholly due to rotation, we would naively expect rotation velocities to become more important on smaller scales. The interferometric PV diagrams and channel maps of these systems reveal smallscale kinematic detail invisible in single-dish data but are also sensitive to emission on size scales between 1000 and $10,000 \mathrm{AU}$. On this scale, differences between the various model PV diagrams shown in Figures 2 and 3 become apparent. The sources presented in this paper represent some of the most well ordered systems kinematically and morphologically from Paper II. However, in spite of this, there are still difficulties in disentangling rotation versus infall in the velocity fields and PV diagrams, which must be recognized when using data of this kind to test theories of star formation.

\subsection{Velocity Gradients as Rotation}

It was shown in Paper II that the envelopes often have substantial velocity gradients $\left(\sim 2.3 \mathrm{~km} \mathrm{~s}^{-1} \mathrm{pc}^{-1}\right)$ out to $10,000 \mathrm{AU}$ and beyond, measured by single-dish mapping. The majority of the envelopes in the sample also had velocity gradients that were within $45^{\circ}$ of normal to the outflow direction, reinforcing an interpretation as rotation. Previous work has often made the assumption that the envelope velocity profile reflects solid-body rotation, even at high resolution (e.g., Chen et al. 2007). The protostars L1165, CB 230, and L1157 are incon- sistent with solid-body rotation with their constant velocities out to large scales. Therefore, if the line-center velocities reflect rotation, then it must be a differential rotation curve with non-constant angular momentum; constant angular momentum would yield $v \propto R^{-1}$. HH 211 specifically could be consistent with solid-body rotation, and IRAS 16253-2429 could also be represented by solid-body rotation but not uniquely. If the characteristic large-scale velocity gradient for $\mathrm{HH} 211$ from Paper II $\left(6.9 \mathrm{~km} \mathrm{~s}^{-1} \mathrm{pc}^{-1}\right)$ is interpreted as rotation, the inferred centrifugal radii of this material are $\sim 25,000$ AU by applying Equation (5). This assumes that material from 10,000 AU fell in to $R_{C}$, with a $0.5 M_{\odot}$ central object. However, this calculation assumes a constant angular velocity for the entire envelope.

With the interferometer and single-dish data from Paper II, we can measure the line-center velocity from the inner to outer envelope at many positions and calculate the centrifugal radius of material throughout the envelope. We have calculated $R_{C}$ versus radius from the observed line-center velocities, assuming a central object mass of $0.5 M_{\odot}$ and that the observed velocities reflect rotation. The results in Figure 15 show that inside of $\sim 5000$ AU the centrifugal radii are below 1000 AU. However, on scales greater than $5000 \mathrm{AU}$, the centrifugal radii are in excess of $1000 \mathrm{AU}$, except for IRAS $16253-2429$. HH 211 notably has an unrealistic $R_{C}>10,000 \mathrm{AU}$ at $R \sim 6500 \mathrm{AU}$. All these centrifugal radii are substantially larger than the characteristic sizes of circumstellar disks ( $250 \mathrm{AU}$; Andrews \& Williams 2007; Andrews et al. 2009). We note that $R_{C}$ could be smaller, if the central object masses are larger than assumed. Moreover, if much of the material observed on 10,000 AU scales does become incorporated into the protostar, then the implied centrifugal radii will be smaller by about a factor of three at $R=10,000 \mathrm{AU}$ since the masses at $R<10,000 \mathrm{AU}$ are $\sim 1 M_{\odot}$; however, this does 


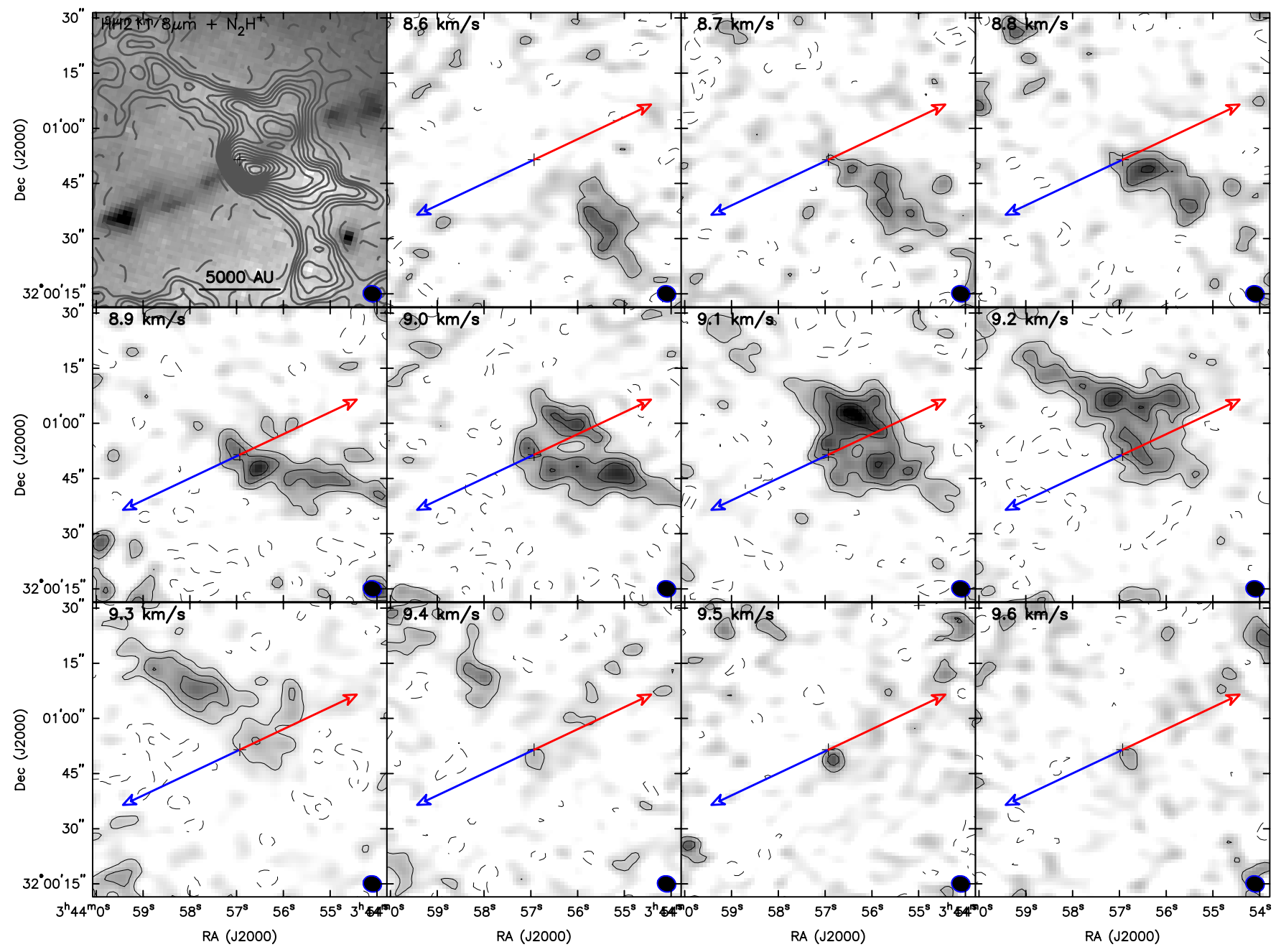

Figure 13. HH 211: channel maps of the isolated $\mathrm{N}_{2} \mathrm{H}^{+}\left(J F_{1} F=101 \rightarrow 012\right)$ line; the blue and red arrows mark the outflow direction, and the cross marks the location of the protostar. The $\mathrm{N}_{2} \mathrm{H}^{+}$emission in the different velocity channels clearly traces a filamentary envelope with a velocity gradient across the protostar that appears fairly linear. Contours in the channel maps start at $\pm 3 \sigma$, increasing in increments of $\pm 3 \sigma$, where $\sigma=0.4 \mathrm{~K}$. The contours in the integrated intensity map are $\pm 10 \sigma, 20 \sigma, \ldots$, where $\sigma=0.149 \mathrm{~K} \mathrm{~km} \mathrm{~s}^{-1}$.

(A color version of this figure is available in the online journal.)

not fully alleviate the problem since corrections will be less at smaller radii.

Rotation of the measured magnitude is expected to cause fragmentation during collapse on large, $\sim 1000 \mathrm{AU}$, scales (Rafikov 2005, 2007; Kratter et al. 2010; Zhu et al. 2012). Of the sources shown, only CB 230 is a wide binary with a separation of $\sim 3000 \mathrm{AU}$. However, the gas kinematics around CB 230 do not indicate rotationally supported motion on this scale, suggesting that the companion did not form via rotational fragmentation. The large centrifugal radii inferred for four of five sources, especially $\mathrm{HH} 211$, may indicate that another dynamical process is contributing to the observed line-center velocities. If the observed velocities are indeed rotation, then to prevent fragmentation, the outer envelopes must be removed before they fall in to the centrifugal radius. Outflows are one way to entrain and evacuate ambient material (Arce \& Sargent 2006); however, it seems unlikely that this mechanism can efficiently work for highly filamentary envelopes that are extended in the direction normal to the outflow. The physical implications of the observed velocities reflecting only rotation seem to suggest that there are additional contributions to the velocity field from dynamical processes other than rotation.

\subsection{Velocity Gradients from Projected Infall}

Since all these systems appear to be gravitationally bound (Paper II), the most likely process that could contribute to the line-center velocity field away from the region of outflow influence is infall. Figure 1 shows that if an envelope is not axisymmetric, then infall could manifest itself in the observed velocity field across the envelope and a velocity gradient normal to the outflow does not necessarily imply rotation (also see Figure 3). The models demonstrate that the radial motion of a globally infalling filamentary envelope can produce detectable velocity shifts on either side of the envelope even with only $\Phi_{0}= \pm 15^{\circ}$ projection angle within the plane of the sky. The velocity shift remains relatively constant on the same scales that we see in our observations. Moreover, if the envelope is only marginally resolved (as in the single-dish observations), such a velocity shift would likely present itself as a linear gradient in lower resolution single-dish data.

CB 230, L1157, and L1165 all have velocities that are approximately constant between $R \sim 1500$ and 10,000 AU in the PV plots (Figures 5, 7, and 10; Table 2). The PV structures agree with the predicted kinematic structure for an infalling filament and are inconsistent with what would be expected from 




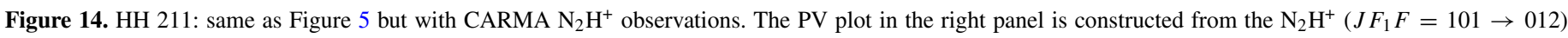

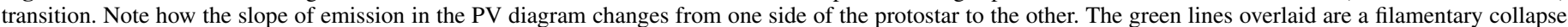

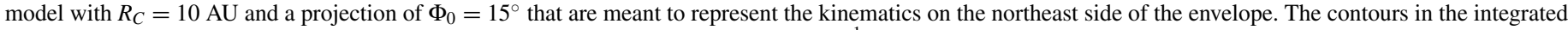

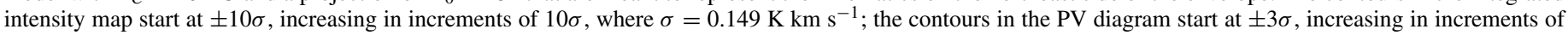
$3 \sigma$, where $\sigma=0.2 \mathrm{~K}$.

(A color version of this figure is available in the online journal.)

axisymmetric collapse or solid-body rotation. The resemblance of the PV plots to the predictions of the infalling filament model, as well as the filamentary morphology of the envelopes, leads us to suggest that the principal dynamic process producing the velocity gradients is large-scale infall and not rotation. However, IRAS 16253-2429 could be described by any of the kinematic models, and HH 211 appears most similar to rapid solid-body rotation, but the non-constant gradient and overly large centrifugal radii may indicate that something more complex is taking place.

The results from the analysis of these data lead us to conclude that many of the large-scale velocity gradients observed in Paper II and other studies most likely arise primarily from infall velocities being projected along our line of sight, with rotation being a subordinate contribution to the velocity field. Furthermore, we suggest that even systems that appear to have linear velocity gradients on large scales probably do not reflect rotation, given the results for $\mathrm{HH} 211$. The linear velocity gradients probably arise from complex projection effects and the formation of cores in a turbulent medium.

Evidence for large-scale infall has been previously seen in the starless core L1544 (Tafalla et al. 1998) and possibly NGC 1333 IRAS 4B (Di Francesco et al. 2001; Jørgensen et al. 2007). Moreover, radiative transfer modeling of the blueasymmetric line profiles of optically thick tracers indicates that infall radii are often of order 5000 AU (Walker et al. 1986; Zhou et al. 1993; Ward-Thompson \& Buckley 2001; Narayanan et al. 2002). Furthermore, starless cores forming in the filamentary dark cloud L1517 appear to have material flowing toward the dense cores (Hacar \& Tafalla 2011) as viewed in the line-center velocities of the filaments. The large-scale flow of material toward the starless cores embedded within filaments is consistent with our picture of the envelopes infalling from large scales in these protostellar systems. We may be seeing the later evolution of this process in our sample, while Hacar \& Tafalla (2011) are seeing the beginning.

The possibility of projected infall makes the true rotation velocities difficult to disentangle in protostellar envelopes with complex morphological structure, limiting the ability to definitively measure angular momentum at large scales. Rotation must be measured at smaller scales, where the rotation velocity will be larger owing to conservation of angular momentum during collapse.

\subsection{Effects of Extended Mass}

The CMU infall model assumes a dominant central mass in the calculation of the velocity field. However, there is substantial mass on scales larger than $\sim 1000 \mathrm{AU}$ in all sources that could certainly affect the kinematics observed. We can simplistically characterize the effects of extended mass by employing Gauss's law for gravity, in which any mass interior to it will appear as a point mass and the free-fall velocity is

$$
v_{\mathrm{ff}}=\left(\frac{2 G M_{\mathrm{enc}}}{R}\right)^{1 / 2} .
$$

Since $M \propto \rho(R) R^{3}$, we can rewrite the free-fall velocity with the following proportionality:

$$
v_{\mathrm{ff}} \propto \rho(R)^{1 / 2} R
$$

Thus, an object with a spherically averaged density profile of $\rho(R) \propto R^{-2}$ will have $v_{\mathrm{ff}} \sim$ constant out to large scales. Most of the objects examined in this paper have approximately constant velocities on large scales, strongly suggesting that they are infalling and extended mass is influencing their kinematics. 

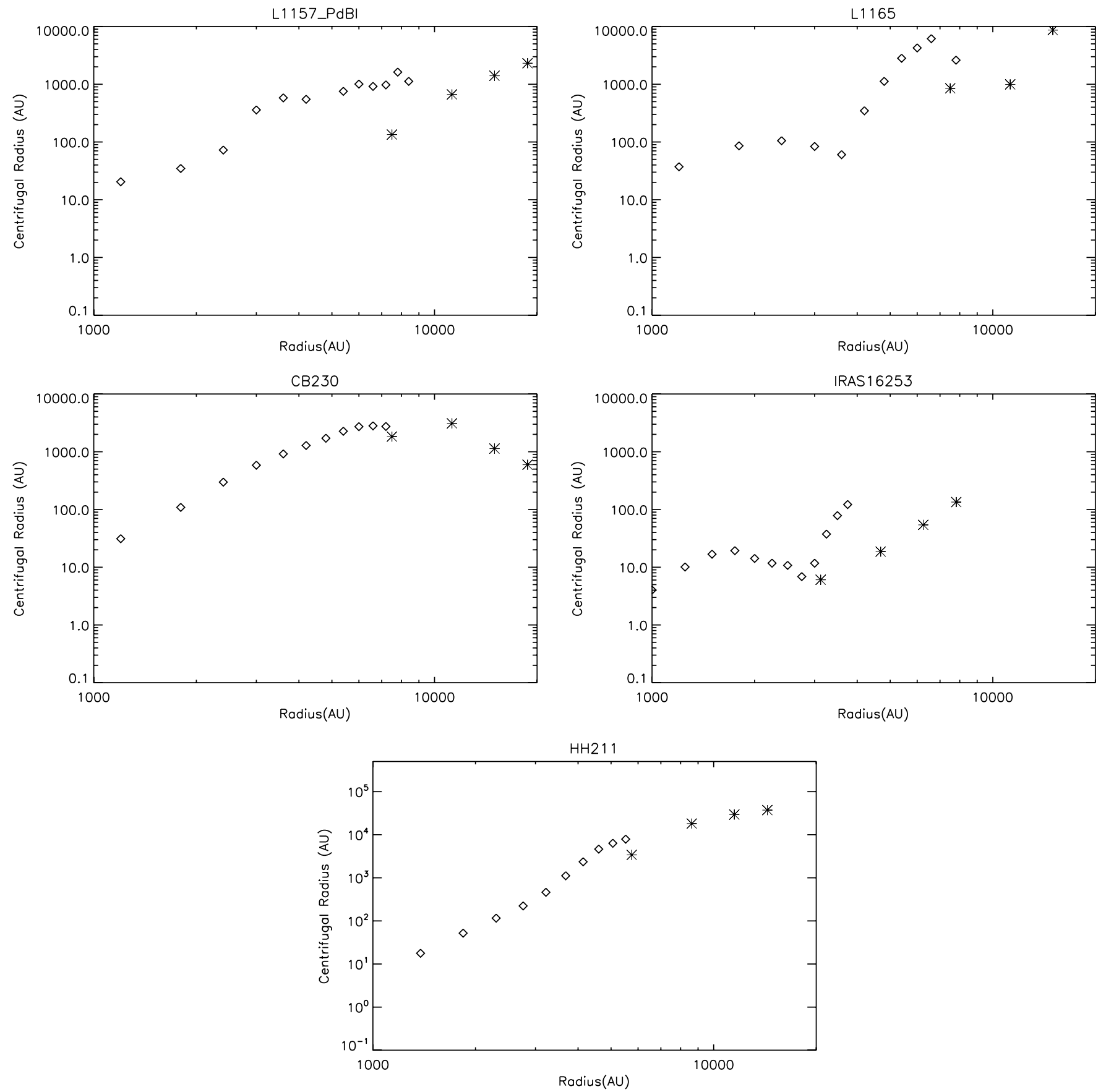

Figure 15. Plot of centrifugal radius $\left(R_{C}\right)$ vs. radius for L1157, L1165, CB 230, IRAS 16253-2429, and HH 211 assuming that the observed velocities reflect rotation. $R_{C}$ is calculated from the observed line-center velocities from Paper II and converted to a centrifugal radius using the relation $R_{C}=R^{2}\left(V-V_{\mathrm{lsr}}\right)^{2} / G M(\mathrm{Equation}(5))$, where $M=0.5 M_{\odot}$ is assumed.

Even with significant extended mass, the predictions of the filament model remain valid; extended mass simply increases the projected infall velocities on large scales, such that they do not approach zero as quickly. In the axisymmetric case, the extended mass would make the infall velocities remain quite broad on large scales, inconsistent with the observations. Lastly, projected infall could mimic the signature of solid-body rotation if the density were constant with increasing radius; however, on the scales of the protostellar envelopes $(\sim 0.1 \mathrm{pc})$ there does not appear to be a region of constant density in the molecular line emission or $8 \mu \mathrm{m}$ extinction. Nevertheless, this effect could be evident in larger scale molecular clouds such as those observed by Arquilla \& Goldsmith (1986).

\subsection{Linewidth Constraints on Infall and Morphology}

Evidence for large-scale infall is also found in the linewidths of the envelopes. The $\mathrm{N}_{2} \mathrm{H}^{+}$and $\mathrm{NH}_{3}$ linewidths in protostellar envelopes are often observed to be about a factor of two or three larger than expected for purely thermal linewidths $\left(0.13 \mathrm{~km} \mathrm{~s}^{-1}\right.$ for $\mathrm{N}_{2} \mathrm{H}^{+}$and $0.22 \mathrm{~km} \mathrm{~s}^{-1}$ for $\mathrm{NH}_{3}$ ) for $T=10 \mathrm{~K}$ gas (e.g., Caselli et al. 2002; Chen et al. 2007; Paper II). Infall motions themselves on large scales, in an axisymmetric envelope, would cause substantial line broadening $\left(\sim 0.5 \mathrm{~km} \mathrm{~s}^{-1}\right)$ owing to the superposition of radially infalling material along the line of sight (Figure 2). The line broadening generated by the infalling motions of filamentary envelopes in Figure 3 is often just 
$0.2 \mathrm{~km} \mathrm{~s}^{-1}$ between radii of $2000 \mathrm{AU}$ and 10,000 AU, which is consistent with what is observed in the PV plots and in the linewidth fields presented in Paper II. Thus, large-scale infall of axisymmetric or non-axisymmetric protostellar envelopes could naturally give rise to the observed line broadening, and it would not be necessary to invoke a turbulent velocity component to explain the observed linewidths.

\subsection{Comparison with Simulations}

Simulations of protostellar core/envelope formation within large-scale molecular clouds also support the idea that rotation is not being accurately probed by line-center velocity maps. Burkert \& Bodenheimer (2000) showed that for individual cores, the line-center velocity gradient cannot be assumed to probe the angular momentum for individual cores; only the distribution of angular momenta can be derived. More recently, Dib et al. (2010) found that angular momenta from cores estimated from the line-center velocity field versus three-dimensional velocities are overestimated by a factor of $\sim 10$. However, they only remark that the difference arises from the line-center method fitting a global linear gradient and the three-dimensional angular momentum including contributions from "complex dynamical behavior," which could be taken to mean turbulence and/or infall. Finally, Smith et al. (2011) investigated the collapse of filamentary cores in their global simulation, finding that there is infall from larger scales toward the cores themselves. Furthermore, a recent detailed analysis of the velocity fields shows significant complexity with contributions from infall, turbulence, and some net rotation (R. J. Smith et al. 2012, in preparation). Realistic simulations of collapse have the promise to enable a better understanding of the net angular momentum in cores as they will enable statistical comparisons with observational data, but with the ability to differentiate rotation and infall.

\section{SUMMARY AND CONCLUSIONS}

We have presented an analysis of the dynamical processes giving rise to the observed kinematic structure in protostellar envelopes. This was done by comparing interferometric $\mathrm{N}_{2} \mathrm{H}^{+}$ and $\mathrm{NH}_{3}$ channel maps and $\mathrm{PV}$ diagrams with our qualitative expectations for envelope kinematics and the predicted kinematics of collapse models. We have constructed an analytic model that approximates filamentary infall, based on the rotating collapse model (Ulrich 1976; Cassen \& Moosman 1981), enabling us to explore projection effects in a simple non-axisymmetric system. We have matched these models to the observations in order to determine the likelihood of rotation or infall in an axisymmetric or filamentary envelope producing the observed velocity structure. The PV diagrams of the infalling axisymmetric and filamentary models are generally distinct from each other on scales between 1000 and 10,000 AU. The models of filamentary collapse viewed at different projection angles show that infall motions along the long axis of a protostellar envelope can produce systematic velocity shifts across the protostar without the need for significant rotation at large scales. These velocity shifts when viewed at low resolution in single-dish measurements would give the appearance of an approximately linear velocity gradient.

The velocity structures of L1157, CB 230, and L1165 are found to be inconsistent with uniform rotation and tend to have constant velocity outside the inner envelope. If the envelope velocities reflected rotation, then the centrifugal radii calculated on scales larger than $5000 \mathrm{AU}$ would be $>1000 \mathrm{AU}$, larger than a typical circumstellar disk. The envelope of HH 211 has a velocity structure consistent with solid-body rotation; however, the envelope is a complex filament and the implied centrifugal radii are unrealistically large. IRAS 16253-2429 has the most symmetric, spherical envelope in the sample and also has very little velocity structure, making all the kinematic models degenerate; however, if rotation is assumed, the implied centrifugal radii are reasonable for this object.

Given that the kinematics of L1157, CB 230, and L1165 seem to reflect projected infall and HH 211 likely has some infall component to its velocities, we therefore suggest the following scenario. The protostellar envelopes we have observed are in a state of global collapse, and the projection of the infall velocities along the filamentary envelopes gives rise to the velocity distributions observed in the PV plots and moment maps in Paper II. This suggests that envelopes with greater degrees of morphological complexity should have similarly complex velocity fields resulting from projected infall velocities.

While there must certainly be net rotation in these envelopes, the degree to which it is solid body is uncertain, and global simulations indicate that the turbulent medium from which the cores formed would impart net angular momentum to the envelope. While we cannot unequivocally prove that our observations are probing infall rather than rotation, we have shown that it is a viable interpretation in asymmetric systems. Moreover, the possibility of infall velocity components in linecenter velocity fields indicates that measurements of angular momentum on large scales can be highly inaccurate. To more definitively measure angular momentum, one must look on small scales where conservation of angular momentum may make the rotation velocities larger and more apparent.

We thank the anonymous referee for a thoughtful review, improving the clarity of the final manuscript. The authors also wish to thank A. Goodman, R. Smith, S. Offner, and M. Borkin for useful discussions. We wish to thank the CARMA observers for carrying out the observations. We thank V. Piétu for assistance with the PdBI data reduction. Support for CARMA construction was derived from the states of Illinois, California, and Maryland, the James S. McDonnell Foundation, the Gordon and Betty Moore Foundation, the Kenneth T. and Eileen L. Norris Foundation, the University of Chicago, the Associates of the California Institute of Technology, and the National Science Foundation. Ongoing CARMA development and operations are supported by the National Science Foundation under a cooperative agreement, and by the CARMA partner universities. The National Radio Astronomy Observatory is a facility of the National Science Foundation operated under cooperative agreement by Associated Universities, Inc. IRAM is supported by INSU/CNRS (France), MPG (Germany), and IGN (Spain). J. J. Tobin acknowledges support from HST-GO-11548.04-A, the University of Michigan Rackham Dissertation Fellowship, Spitzer archival research program 50668, and Hubble Fellowship grant HF-51300.01-A awarded by the Space Telescope Science Institute, which is operated by the Association of Universities for Research in Astronomy, Inc., for NASA, under contract NAS 5-26555.

Facilities: CARMA, VLA, IRAM: Interferometer, Spitzer (IRAC)

\section{REFERENCES}

Andrews, S. M., \& Williams, J. P. 2007, ApJ, 659, 705

Andrews, S. M., Wilner, D. J., Hughes, A. M., Qi, C., \& Dullemond, C. P. 2009, ApJ, 700, 1502 
Arce, H. G., \& Sargent, A. I. 2006, ApJ, 646, 1070

Arquilla, R., \& Goldsmith, P. F. 1986, ApJ, 303, 356

Bacmann, A., André, P., Puget, J., et al. 2000, A\&A, 361, 555

Bate, M. R., Bonnell, I. A., \& Bromm, V. 2003, MNRAS, 339, 577

Belloche, A., \& André, P. 2004, A\&A, 419, L35

Belloche, A., André, P., Despois, D., \& Blinder, S. 2002, A\&A, 393, 927

Benson, P. J., \& Myers, P. C. 1989, ApJS, 71, 89

Boss, A. P. 1995, RevMexAA, 1, 165

Brinch, C., Crapsi, A., Jørgensen, J. K., Hogerheijde, M. R., \& Hill, T. 2007, A\&A, 475, 915

Burkert, A., \& Bodenheimer, P. 1993, MNRAS, 264, 798

Burkert, A., \& Bodenheimer, P. 2000, ApJ, 543, 822

Caselli, P., Benson, P. J., Myers, P. C., \& Tafalla, M. 2002, ApJ, 572, 238

Cassen, P., \& Moosman, A. 1981, Icarus, 48, 353

Chen, X., Launhardt, R., \& Henning, T. 2007, ApJ, 669, 1058

Chevalier, R. A. 1983, ApJ, 268, 753

Chiang, H., Looney, L. W., Tobin, J. J., \& Hartmann, L. 2010, ApJ, 709, 470

Dib, S., Hennebelle, P., Pineda, J. E., et al. 2010, ApJ, 723, 425

Di Francesco, J., Myers, P. C., Wilner, D. J., Ohashi, N., \& Mardones, D. 2001, ApJ, 562, 770

Enoch, M. L., Evans, N. J., Sargent, A. I., \& Glenn, J. 2009, ApJ, 692, 973

Foster, P. N., \& Chevalier, R. A. 1993, ApJ, 416, 303

Froebrich, D. 2005, ApJS, 156, 169

Goodman, A. A., Benson, P. J., Fuller, G. A., \& Myers, P. C. 1993, ApJ, 406, 528

Gueth, F., Guilloteau, S., Dutrey, A., \& Bachiller, R. 1997, A\&A, 323, 943

Hacar, A., \& Tafalla, M. 2011, A\&A, 533, A34

Hartmann, L. (ed.) 2009, Accretion Processes in Star Formation: Second Edition (Cambridge: Cambridge Univ. Press)

Hirota, T., Honma, M., Imai, H., et al. 2011, PASJ, 63, 1

Jørgensen, J. K., Bourke, T. L., Myers, P. C., et al. 2007, ApJ, 659, 479

Kauffmann, J., Bertoldi, F., Bourke, T. L., Evans, N. J., II, \& Lee, C. W. 2008, A\&A, 487, 993

Kirk, J. M., Ward-Thompson, D., Di Francesco, J., et al. 2009, ApJS, 185, 198

Kratter, K. M., Matzner, C. D., Krumholz, M. R., \& Klein, R. I. 2010, ApJ, 708, 1585

Larson, R. B. 1969, MNRAS, 145, 271
Lee, C., Hirano, N., Palau, A., et al. 2009, ApJ, 699, 1584

Lee, J., Bergin, E. A., \& Evans, N. J., II 2004, ApJ, 617, 360

Loinard, L., Torres, R. M., Mioduszewski, A. J., \& Rodríguez, L. F. 2008, ApJ, 675, L29

Myers, P. C. 2005, ApJ, 623, 280

Myers, P. C., Fuller, G. A., Goodman, A. A., \& Benson, P. J. 1991, ApJ, 376 561

Narayanan, G., Moriarty-Schieven, G., Walker, C. K., \& Butner, H. M. 2002, ApJ, 565, 319

Rafikov, R. R. 2005, ApJ, 621, L69

Rafikov, R. R. 2007, ApJ, 662, 642

Regos, E., \& Geller, M. J. 1989, AJ, 98, 755

Ryden, B. S. 1996, ApJ, 471, 822

Shirley, Y. L., Evans, N. J., II, Rawlings, J. M. C., \& Gregersen, E. M 2000, ApJS, 131, 249

Shu, F. H. 1977, ApJ, 214, 488

Shu, F. H., Adams, F. C., \& Lizano, S. 1987, ARA\&A, 25, 23

Smith, R. J., Glover, S. C. O., Bonnell, I. A., Clark, P. C., \& Klessen, R. S. 2011, MNRAS, 411, 1354

Stutz, A. M., Rieke, G. H., Bieging, J. H., et al. 2009, ApJ, 707, 137

Tafalla, M., Mardones, D., Myers, P. C., et al. 1998, ApJ, 504, 900

Tanner, J. D., \& Arce, H. G. 2011, ApJ, 726, 40

Terebey, S., Shu, F. H., \& Cassen, P. 1984, ApJ, 286, 529

Tobin, J. J., Hartmann, L., Chiang, H.-F., et al. 2011, ApJ, 740, 45

Tobin, J. J., Hartmann, L., Looney, L. W., \& Chiang, H. 2010, ApJ, 712, 1010

Ulrich, R. K. 1976, ApJ, 210, 377

Visser, A. E., Richer, J. S., \& Chandler, C. J. 2002, AJ, 124, 2756

Volgenau, N. H., Mundy, L. G., Looney, L. W., \& Welch, W. J. 2006, ApJ, 651, 301

Walker, C. K., Lada, C. J., Young, E. T., Maloney, P. R., \& Wilking, B. A. 1986, ApJ, 309, L47

Ward-Thompson, D., \& Buckley, H. D. 2001, MNRAS, 327, 955

Young, K. E., Enoch, M. L., Evans, N. J., II, et al. 2006, ApJ, 644, 326

Zhou, S. 1992, ApJ, 394, 204

Zhou, S., Evans, N. J., II, Koempe, C., \& Walmsley, C. M. 1993, ApJ, 404, 232

Zhu, Z., Hartmann, L., \& Gammie, C. 2010, ApJ, 713, 1143

Zhu, Z., Hartmann, L., Nelson, R. P., \& Gammie, C. F. 2012, ApJ, 746, 110 